\title{
ARBITRARY LAGRANGIAN-EULERIAN HYBRIDIZABLE DISCONTINUOUS GALERKIN METHODS FOR INCOMPRESSIBLE FLOW WITH MOVING BOUNDARIES AND INTERFACES
}

\author{
GUOSHENG FU
}

\begin{abstract}
We present a class of Arbitrary Lagrangian-Eulerian hybridizable discontinuous Galerkin methods for the incompressible flow with moving boundaries and interfaces including two-phase flow with surface tension.
\end{abstract}

\section{INTRODUCTION}

Incompressible flow problems with moving boundaries and interfaces appear naturally in a number of practical applications such as free surface flow, multi-phase flow, and fluid-structure interactions (FSI) 22, 45]. Using the terminology and categorization used in [50, a method for flows with moving boundaries can be an interface-tracking (boundary-fitted) method or an interface-capturing (non-boundary-fitted) method, or possibly a combination of the two. Examples of boundary-fitted methods include the arbitrary Lagrangian-Eulerian (ALE) method [11, 12, 28] and the space-time method [36, 51,52,54, examples of non-boundary-fitted methods include the immersed boundary method 37, 41, the immersed finite element method [35, 58], the fictitious domain method [21], and the extended/generalized finite element method [6,15], among many others.

In this paper, we present novel finite element methods for incompressible flow with moving boundaries and interfaces using the ALE framework. Our spatial discretization is based on a novel hybridizable discontinuous Galerkin (HDG) formulation that may produce an exactly divergencefree velocity approximation; see 77 for a review of HDG methods. HDG methods that yield an exactly divergence-free velocity approximation have been extensively studied for incompressible flow on static meshes, see, e.g., $10,19,31,34,44$. The extension of these schemes to moving domain problems was recently studied by Horvath and Rhebergen [25,26] within the space-time framework, and by Neunteufel [38] within the ALE framework. In this paper, we present a novel ALE-HDG scheme that is computationally more efficient than those in [25, 26, 38 due to the use of a novel set of hybrid unknowns, which includes the tangential component of velocity $(T V)$ and normalnormal component of the stress $(N N S)$ on the mesh skeleton. We name the new HDG scheme the TVNNS-HDG scheme.

ALE-based finite element methods have been widely used for moving interface incompressible flow problems, see the recent review [53]. The first ALE-based HDG method was introduced in 2016 by Sheldon et. al. [49] for FSI problems. However, the spatial discretization for fluids in [49] was based on the HDG scheme [39] for Stokes flow which does not produce an exactly divergence-free velocity approximation. In 2017, Neunteufel [38] introduced the ALE-H(div)-conforming-HDG method for moving domain incompressible flows and FSI in his diploma thesis. However, due to the use of Piola mapping needed for the $H(\mathrm{div})$-conforming velocity finite element space, the ALE- $H(\mathrm{div})$ conforming-HDG formulation [38] is significantly more complex than ALE schemes based on classical continuous-velocity-based finite element methods like the Taylor-Hood element. In this paper, we present a novel HDG scheme termed TVNNS-HDG that is based on completely discontinuous finite element spaces using the standard pull-back mappings, which produces a divergence-conforming

1991 Mathematics Subject Classification. 65N30, 65N12, 76S05, 76D07.

Key words and phrases. ALE, HDG, divergence-free, moving domain, two-phase flow, surface tension. 
and divergence-free velocity approximation when the underlying mesh consists of affine simplices. The $H$ (div)-conformity of the scheme is achieved via the classical hybridization technique [1, 8], where we first relax the $H$ (div)-conformity of the velocity space then weakly impose it back via a Lagrange multiplier (which is the normal-normal-stress variable). Our spatial discretization on conforming affine simplicial meshes is mathematically equivalent to the divergence-free HDG scheme proposed back in 2010 by Lehrenfeld [33]. Hence, on static conforming simplicial meshes, it readily enjoys properties such as high order accuracy and optimal convergence, important global and local conservation properties, energy-stability, pressure robustness, a minimal amount of numerical dissipation and computational efficiency [34,47].

The rest of the paper is organized as follows. In Section 2, we first introduce the TVNNS-HDG scheme for the steady-state incompressible Stokes equations, then apply it to the moving domain Navier-Stokes equations using the ALE framework. In Section 3, we extend the ALE-TVNNS-HDG scheme to incompressible two-phase flow with surface tension. Numerical results are presented in Section 4. We conclude in Section 5 .

\section{The ALE-TVNNS-HDG Scheme For moving Domain NAVIER-Stokes EQUations}

2.1. The ALE-Navier-Stokes equations. Consider the Navier-Stokes equation on a smoothvarying moving domain $\Omega^{t} \subset \mathbb{R}^{d}, d \in\{2,3\}$, for $t \in[0, T]$, given by a smooth ALE map [40, 42]:

$$
\mathcal{A}_{t}: \Omega^{0} \subset \mathbb{R}^{d} \longrightarrow \Omega^{t}, \quad \mathbf{x}\left(\mathbf{x}_{0}, t\right)=\mathcal{A}_{t}\left(\mathbf{x}_{0}\right), \quad \forall t \in[0, T]
$$

where the initial configuration $\Omega^{0}$ at $t=0$ is considered as the reference configuration. The NavierStokes equations in ALE non-conservative form [40,42] is given as follows:

$$
\begin{array}{rr}
\left.\rho \frac{\partial \boldsymbol{u}}{\partial t}\right|_{\mathbf{x}_{0}}+\rho(\boldsymbol{u}-\boldsymbol{\omega}) \cdot \nabla_{\mathbf{x}} \boldsymbol{u}-\operatorname{div}_{\mathbf{x}}\left(2 \mu \mathbf{D}_{\mathbf{x}}(\boldsymbol{u})-p \boldsymbol{I}\right)=\rho \boldsymbol{f}, & \text { in } \Omega^{t} \times[0, T] \\
\operatorname{div}_{\mathbf{x}} \boldsymbol{u}=0, & \text { in } \Omega^{t} \times[0, T]
\end{array}
$$

where $\mathbf{D}_{\mathbf{x}}$ is the symmetric strain rate tensor

$$
\mathbf{D}_{\mathbf{x}}(\boldsymbol{u})=\frac{1}{2}\left(\nabla_{\mathbf{x}} \boldsymbol{u}+\left(\nabla_{\mathbf{x}} \boldsymbol{u}\right)^{T}\right),
$$

$\boldsymbol{I}$ is the identity tensor, $\boldsymbol{u}(\mathbf{x}, t)$ is the velocity field, $p(\mathbf{x}, t)$ is the pressure, $\rho$ is the (constant) fluid density, $\mu$ is the (constant) coefficient of dynamic viscosity, $\boldsymbol{f}$ is the body forces, and

$$
\boldsymbol{\omega}(\mathbf{x}, t)=\left.\frac{\partial \mathbf{x}}{\partial t}\right|_{\mathbf{x}_{0}}=\frac{\partial \mathcal{A}_{t}}{\partial t} \circ \mathcal{A}_{t}^{-1}(\mathbf{x})
$$

denotes the domain velocity. Throughout this section, we assume that ALE map (1) is given, although in most applications it represents a further unknown of the problem.

2.2. The TVNNS-HDG scheme for the steady-state Stokes equations. In order to present our HDG discretization for the equations (2), we first present the TVNNS-HDG scheme for the following steady-state Stokes equations on the domain $\Omega^{t}$, with a fixed time $t \in[0, T]$ :

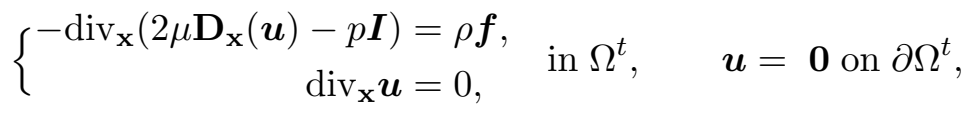

where the stress $\boldsymbol{\sigma}:=2 \mu \mathbf{D}_{\mathbf{x}}(\boldsymbol{u})-p \boldsymbol{I}$. We assume that the domain $\Omega^{t}$ is obtained from the reference domain $\Omega^{0}$ by the mapping (1). Let $\mathcal{T}_{h}^{0}:=\left\{T^{0}\right\}$ be a conforming simplicial triangulation of $\Omega^{0}$, and let $\mathcal{E}_{h}^{0}$ be the set of facets of $\mathcal{T}_{h}^{0}$. We denote $\mathcal{T}_{h}^{t}:=\left\{\mathcal{A}_{t}\left(T^{0}\right)\right\}$ as the mapped triangulation on the domain $\Omega^{t}$, and denote $\mathcal{E}_{h}^{t}$ as the set of facets of $\mathcal{T}_{h}^{t}$. We set $h$ to be the maximum mesh size of $\mathcal{T}_{h}^{t}$. Given an affine simplex $\widehat{S} \subset \mathbb{R}^{d}, d=1,2,3$, we denote $\mathcal{P}^{m}(\widehat{S})$ as the space of polynomials of 
degree at most $m$ on the mesh for any integer $m \geq 0$. Given any mapped simplex $S=\mathcal{A}(\widehat{S})$, with an abuse of notation, we also denote

$$
\mathcal{P}^{m}(S):=\left\{v \in L^{2}(S): \quad v=\widehat{v} \circ \mathcal{A}^{-1} \text { with }\left.\widehat{v}\right|_{\widehat{S}} \in \mathcal{P}^{k}(\widehat{S})\right\}
$$

Note that the standard pull-back transformation $\bullet(\mathbf{x}, t)=\widetilde{\bullet} \circ \mathcal{A}^{-1}(\mathbf{x})$ is used to define the space $\mathcal{P}^{m}(S)$. We now define the following discontinuous polynomial finite element spaces based on pull-back transformations:

$$
\begin{aligned}
& \boldsymbol{V}_{h}^{t}:=\left\{\boldsymbol{v} \in\left[L^{2}\left(\mathcal{T}_{h}^{t}\right)\right]^{d}:\left.\quad \boldsymbol{v}\right|_{T} \in\left[\mathcal{P}^{k}(T)\right]^{d}, \quad \forall T \in \mathcal{T}_{h}^{t}\right\}, \\
& Q_{h}^{t}:=\left\{q \in L^{2}\left(\mathcal{T}_{h}^{t}\right):\left.\quad q\right|_{T} \in \mathcal{P}^{k-1}(T), \quad \forall T \in \mathcal{T}_{h}^{t}\right\}, \\
& \widehat{\boldsymbol{V}}_{h}^{t}:=\left\{\widehat{\boldsymbol{v}} \in\left[L^{2}\left(\mathcal{E}_{h}^{t}\right)\right]^{d}: \quad \widehat{\boldsymbol{v}}=\oplus_{j=1}^{d-1}\left(\widehat{v}_{j}\right) \boldsymbol{t}_{j}, \text { with }\left.\widehat{v}_{j}\right|_{F} \in \mathcal{P}^{k}(F), \quad \forall F \in \mathcal{E}_{h}^{t}\right\}, \\
& \widehat{\boldsymbol{V}}_{h, 0}^{t}:=\left\{\widehat{\boldsymbol{v}} \in \widehat{\boldsymbol{V}}_{h}^{t}:\left.\quad \widehat{\boldsymbol{v}}\right|_{F}=\mathbf{0}, \quad \forall F \in \mathcal{E}_{h}^{t} \cap \partial \Omega^{t}\right\}, \\
& \widehat{M}_{h}^{t}:=\left\{\widehat{\tau}^{n n} \in L^{2}\left(\mathcal{E}_{h}^{t}\right):\left.\quad \widehat{\tau}^{n n}\right|_{F} \in \mathcal{P}^{k}(F), \quad \forall F \in \mathcal{E}_{h}^{t}\right\},
\end{aligned}
$$

where $\left\{\boldsymbol{t}_{j}\right\}_{j=1}^{d-1}$ are the orthogonal tangential directions on the mesh skeleton $\varepsilon_{h}^{t}$. The weak formulation of the TVNNS-HDG scheme for (3) is given as follows: Find $\left(\boldsymbol{u}_{h}, p_{h}, \widehat{\boldsymbol{u}}_{h}, \widehat{\sigma}_{h}^{n n}\right) \in \boldsymbol{V}_{h}^{t} \times Q_{h}^{t} \times$ $\widehat{\boldsymbol{V}}_{h, 0}^{t} \times \widehat{M}_{h}^{t}$ such that

$$
\begin{aligned}
2 \mu \mathcal{B}_{h}\left(\left(\boldsymbol{u}_{h}, \widehat{\boldsymbol{u}}_{h}\right),\left(\boldsymbol{v}_{h}, \widehat{\boldsymbol{v}}_{h}\right)\right)-\mathcal{D}_{h}\left(\boldsymbol{v}_{h},\left(p_{h}, \widehat{\sigma}_{h}^{n n}\right)\right) & =f_{h}\left(\boldsymbol{v}_{h}\right), \\
\mathcal{D}_{h}\left(\boldsymbol{u}_{h},\left(q_{h}, \widehat{\tau}_{h}^{n n}\right)\right) & =0
\end{aligned}
$$

for all $\left(\boldsymbol{v}_{h}, q_{h}, \widehat{\boldsymbol{v}}_{h}, \widehat{\tau}_{h}^{n n}\right) \in \boldsymbol{V}_{h}^{t} \times Q_{h}^{t} \times \widehat{\boldsymbol{V}}_{h, 0}^{t} \times \widehat{M}_{h}^{t}$, where

$$
\begin{aligned}
& \mathcal{B}_{h}\left(\left(\boldsymbol{u}_{h}, \widehat{\boldsymbol{u}}_{h}\right),\left(\boldsymbol{v}_{h}, \widehat{\boldsymbol{v}}_{h}\right)\right):=\sum_{T \in \mathcal{T}_{h}^{t}} \int_{T} \mathbf{D}_{\mathbf{x}}\left(\boldsymbol{u}_{h}\right): \mathbf{D}_{\mathbf{x}}\left(\boldsymbol{v}_{h}\right) \mathrm{dx}-\int_{\partial T} \mathbf{D}_{\mathbf{x}}\left(\boldsymbol{u}_{h}\right) \boldsymbol{n} \cdot \operatorname{tang}\left(\boldsymbol{v}_{h}-\widehat{\boldsymbol{v}}_{h}\right) \mathrm{ds} \\
&-\underbrace{\int_{\partial T} \mathbf{D}_{\mathbf{x}}\left(\boldsymbol{v}_{h}\right) \boldsymbol{n} \cdot \operatorname{tang}\left(\boldsymbol{u}_{h}-\widehat{\boldsymbol{u}}_{h}\right) \mathrm{d} s}_{\text {for symmetry }}+\underbrace{\int_{\partial T} \frac{\alpha(k+1)^{2}}{h} \operatorname{tang}\left(\boldsymbol{u}_{h}-\widehat{\boldsymbol{u}}_{h}\right) \cdot \operatorname{tang}\left(\boldsymbol{v}_{h}-\widehat{\boldsymbol{v}}_{h}\right) \mathrm{ds}}_{\text {for stability }} \\
& \mathcal{D}_{h}\left(\boldsymbol{u}_{h},\left(q_{h}, \widehat{\tau}_{h}^{n n}\right)\right):=\sum_{T \in \mathcal{T}_{h}^{t}} \int_{T} \operatorname{div}_{\mathbf{x}}\left(\boldsymbol{u}_{h}\right) q_{h} \mathrm{dx}+\int_{\partial T} \boldsymbol{u}_{h} \cdot \boldsymbol{n} \widehat{\tau}_{h}^{n n} \mathrm{ds}, \\
& f_{h}\left(\boldsymbol{v}_{h}\right):=\sum_{T \in \mathcal{T}_{h}^{t}} \int_{T} \rho \boldsymbol{f} \cdot \boldsymbol{v}_{h} \mathrm{dx},
\end{aligned}
$$

where $\boldsymbol{n}$ is the normal direction, $\operatorname{tang}(\boldsymbol{w}):=\boldsymbol{w}-(\boldsymbol{w} \cdot \boldsymbol{n}) \boldsymbol{n}$ denotes the tangential component of a vector $\boldsymbol{w}$, the variable $\widehat{\sigma}_{h}^{n n}$ approximates the normal-normal component of the stress $(\boldsymbol{\sigma} \boldsymbol{n}) \cdot \boldsymbol{n}$, and $\alpha>0$ is a sufficiently large stabilization constant taken to be 4 in all our numerical simulations.

The following result shows that the scheme (5) on affine simplicial meshes is mathematically equivalent to the divergence-free HDG scheme proposed in [33, Equation (2.3.10)]. Hence, optimal (pressure-robust) velocity error estimate that is independent of the pressure regularity can be obtained for the scheme (5), see [33, Lemma 2.3.13-2.3.14].

Theorem 2.1. Assume the conforming simplicial mesh $\mathcal{T}_{h}^{t}$ is affine. Let $\left(\boldsymbol{u}_{h}, p_{h}, \widehat{\boldsymbol{u}}_{h}, \widehat{\sigma}_{h}^{n n}\right) \in \boldsymbol{V}_{h}^{t} \times$ $Q_{h}^{t} \times \widehat{\boldsymbol{V}}_{h, 0}^{t} \times \widehat{M}_{h}^{t}$ be the solution to the scheme (5). Then, $\left(\boldsymbol{u}_{h}, p_{h}, \widehat{\boldsymbol{u}}_{h}\right)$ is the solution to the following divergence-free HDG scheme proposed in [33, Equation (2.3.10)]: Find $\left(\boldsymbol{u}_{h}, p_{h}, \widehat{\boldsymbol{u}}_{h}\right) \in$ 
$\left(\boldsymbol{V}_{h}^{t} \cap H_{0}\left(\operatorname{div}, \Omega^{t}\right)\right) \times Q_{h}^{t} \times \widehat{\boldsymbol{V}}_{h, 0}^{t}$ such that

$$
\begin{aligned}
2 \mu \mathcal{B}_{h}\left(\left(\boldsymbol{u}_{h}, \widehat{\boldsymbol{u}}_{h}\right),\left(\boldsymbol{v}_{h}, \widehat{\boldsymbol{v}}_{h}\right)\right)-\sum_{T \in \mathcal{T}_{h}^{t}} \int_{T} \operatorname{div}_{\mathbf{x}}\left(\boldsymbol{v}_{h}\right) p_{h} \mathrm{dx}=f_{h}\left(\boldsymbol{v}_{h}\right), \\
\sum_{T \in \mathcal{T}_{h}^{t}} \int_{T} \operatorname{div}_{\mathbf{x}}\left(\boldsymbol{u}_{h}\right) q_{h} \mathrm{dx}=0,
\end{aligned}
$$

for all $\left(\boldsymbol{v}_{h}, q_{h}, \widehat{\boldsymbol{v}}_{h}\right) \in\left(\boldsymbol{V}_{h}^{t} \cap H_{0}\left(\operatorname{div}, \Omega^{t}\right)\right) \times Q_{h}^{t} \times \widehat{\boldsymbol{V}}_{h, 0}^{t}$.

Proof. Since the mesh consists of affine simplicies, equation $(5 \mathrm{~b})$ implies that $\boldsymbol{u}_{h}$ is divergenceconforming and divergence-free, and its normal component vanishes on the boundary. Taking test functions $\left(\boldsymbol{v}_{h}, q_{h}, \widehat{\boldsymbol{v}}_{h}\right) \in\left(\boldsymbol{V}_{h}^{t} \cap H_{0}\left(\operatorname{div}, \Omega^{t}\right)\right) \times Q_{h}^{t} \times \widehat{\boldsymbol{V}}_{h, 0}^{t}$ for the scheme (5), we obtain the above equations in Theorem 2.1. This completes the proof.

Remark 2.1. The formulation (5) was briefly mentioned in [34, Remark 7], but it has not been seriously compared with the divergence-conforming velocity space-based formulation [33, 34]. We show in the next subsection that due to the use of completely discontinuous finite element space and pull-back mappings for the velocity approximation, the extension of the scheme (5) to the ALE Navier-Stokes equations (2) poses no extra difficulties.

Remark 2.2. When the mesh $\mathcal{T}_{h}^{t}$ is curved, equation (5b) would neither imply divergence-conformity or locally divergence-free of the velocity approximation $\boldsymbol{u}_{h}$ due to the use of pull-back transformation in (4a) since, in general, $\left.\boldsymbol{u}_{h} \cdot \boldsymbol{n}\right|_{F} \notin \mathcal{P}^{k}(F)$ for curved facets $F$, and $\left.\operatorname{div}_{\mathbf{x}}\left(\boldsymbol{u}_{h}\right)\right|_{T} \notin \mathcal{P}^{k-1}(T)$ for curved elements $T$. The analysis of the scheme (5) for the curved mesh case is more complicated, which consists our ongoing work.

2.3. The ALE-TVNNS-HDG scheme. Here we extend the HDG scheme (5) to the ALE NavierStokes equations (2). In particular, we point out that:

- The nonlinear convection term in (2) will be discretized using a variant 33] of the upwinding technique [9] thanks to the use of discontinuous velocity finite element space, which provides a minimal amount of numerical dissipation that seems to be strong enough to suppress unphysical oscillation and gives the scheme extra stability properties in the convectiondominated case; see, e.g., [17,48. No additional convection stabilization is required for the HDG formulation, which has to be contrasted with methods using continuous velocity approximation, where extra stabilization terms [5] are needed for stability, usually via a variational multiscale approach [27]. We also mention that traditional variational multiscale technique might pollute the divergence-free property of the velocity approximation; see 30, Section 2.2.3], see also the recent work on an isogeometric divergence-free variational multiscale formulation [55].

- Thanks to the use of completely discontinuous velocity finite element space $\boldsymbol{V}_{h}^{t}$ via the pull-back transformation, the time derivative term in (2) can be easily treated similarly as the fixed domain case.

2.3.1. Semidiscrete scheme. For simplicity, we assume the equations $(2)$ is equipped with a homogeneous Dirichlet boundary condition for velocity on the whole boundary $\partial \Omega^{t}$, for all $t \in[0, T]$. Then, the semidiscrete ALE-divergence-free-HDG scheme reads as follows: Find $\left(\boldsymbol{u}_{h}, p_{h}, \widehat{\boldsymbol{u}}_{h}, \widehat{\sigma}_{h}^{n n}\right) \in$ 
$\boldsymbol{V}_{h}^{t} \times Q_{h}^{t} \times \widehat{\boldsymbol{V}}_{h, 0}^{t} \times \widehat{M}_{h}^{t}$ such that

$$
\begin{aligned}
\rho \mathcal{M}_{h}\left(\left.\frac{\partial \boldsymbol{u}_{h}}{\partial t}\right|_{\mathbf{x}_{0}}, \boldsymbol{v}_{h}\right)+\rho \mathcal{C}_{h}\left(\left(\boldsymbol{\omega}, \boldsymbol{u}_{h}, \widehat{\boldsymbol{u}}_{h}\right),\left(\boldsymbol{v}_{h}, \widehat{\boldsymbol{v}}_{h}\right)\right) \\
+2 \mu \mathcal{B}_{h}\left(\left(\boldsymbol{u}_{h}, \widehat{\boldsymbol{u}}_{h}\right),\left(\boldsymbol{v}_{h}, \widehat{\boldsymbol{v}}_{h}\right)\right)-\mathcal{D}_{h}\left(\boldsymbol{v}_{h},\left(p_{h}, \widehat{\sigma}_{h}^{n n}\right)\right)=f_{h}\left(\boldsymbol{v}_{h}\right), \\
\mathcal{D}_{h}\left(\boldsymbol{u}_{h},\left(q_{h}, \widehat{\tau}_{h}^{n n}\right)\right)=0,
\end{aligned}
$$

for all $\left(\boldsymbol{v}_{h}, q_{h}, \widehat{\boldsymbol{v}}_{h}, \widehat{\tau}_{h}^{n n}\right) \in \boldsymbol{V}_{h}^{t} \times Q_{h}^{t} \times \widehat{\boldsymbol{V}}_{h, 0}^{t} \times \widehat{M}_{h}^{t}$, where the mass operator

$$
\mathcal{M}_{h}\left(\boldsymbol{u}_{h}, \boldsymbol{v}_{h}\right):=\sum_{T \in \mathcal{T}_{h}^{t}} \int_{T} \boldsymbol{u}_{h} \cdot \boldsymbol{v}_{h} \mathrm{dx}
$$

and the nonlinear convection operator $\mathcal{C}_{h}\left(\left(\boldsymbol{\omega}, \boldsymbol{u}_{h}, \widehat{\boldsymbol{u}}_{h}\right),\left(\boldsymbol{v}_{h}, \widehat{\boldsymbol{v}}_{h}\right)\right)$ is given below:

$$
\mathcal{C}_{h}:=\sum_{T \in \mathcal{T}_{h}^{t}}-\int_{T} \boldsymbol{u}_{h} \cdot \operatorname{div}_{\mathbf{x}}\left(\left(\boldsymbol{u}_{h}-\boldsymbol{\omega}\right) \otimes \boldsymbol{v}_{h}\right) \mathrm{dx}+\int_{\partial T}\left(\boldsymbol{u}_{h}-\boldsymbol{\omega}\right) \cdot \boldsymbol{n} \widehat{\boldsymbol{u}}_{h}^{u p} \cdot \operatorname{tang}\left(\boldsymbol{v}_{h}-\widehat{\boldsymbol{v}}_{h}\right) \mathrm{ds},
$$

with $\widehat{\boldsymbol{u}}_{h}^{u p}$ is the following upwinding numerical flux for the tangential velocity component:

$$
\widehat{\boldsymbol{u}}_{h}^{u p}:=\left\{\begin{array}{ll}
\operatorname{tang}\left(\boldsymbol{u}_{h}\right) & \text { if }\left(\boldsymbol{u}_{h}-\boldsymbol{\omega}\right) \cdot \boldsymbol{n} \geq 0 \\
\operatorname{tang}\left(\widehat{\boldsymbol{u}}_{h}\right) & \text { if }\left(\boldsymbol{u}_{h}-\boldsymbol{\omega}\right) \cdot \boldsymbol{n}<0
\end{array} .\right.
$$

The ALE Navier-Stokes equations (2) hold the following energy identity:

$$
\frac{1}{2} \frac{d}{d t} \rho\|\boldsymbol{u}\|_{L^{2}\left(\Omega^{t}\right)}^{2}+2 \mu\left\|\mathbf{D}_{\mathbf{x}}(\boldsymbol{u})\right\|_{L^{2}\left(\Omega^{t}\right)}^{2}=\rho \int_{\Omega^{t}} \boldsymbol{f} \cdot \boldsymbol{u} \mathrm{dx} .
$$

The following result shows that a similar energy identity holds for the semidiscrete scheme (7) provided that the mesh is affine.

Theorem 2.2. Assume mesh $\mathcal{T}_{h}^{t}$ is affine. Let $\left(\boldsymbol{u}_{h}, p_{h}, \widehat{\boldsymbol{u}}_{h}, \widehat{\sigma}_{h}^{n n}\right) \in \boldsymbol{V}_{h}^{t} \times Q_{h}^{t} \times \widehat{\boldsymbol{V}}_{h, 0}^{t} \times \widehat{M}_{h}^{t}$ be the solution to the equations (7) for all $t \in[0, T]$. Then the following energy identity holds

$$
\frac{1}{2} \frac{d}{d t} \rho\left\|\boldsymbol{u}_{h}\right\|_{L^{2}\left(\Omega^{t}\right)}^{2}+2 \mu \mathcal{B}_{h}\left(\left(\boldsymbol{u}_{h}, \widehat{\boldsymbol{u}}_{h}\right),\left(\boldsymbol{u}_{h}, \widehat{\boldsymbol{u}}_{h}\right)\right)+\mathrm{DISP}=f_{h}\left(\boldsymbol{u}_{h}\right)
$$

where DISP is the following nonnegative numerical dissipation term:

$$
\mathrm{DISP}:=\frac{1}{2} \rho \sum_{T \in \mathcal{T}_{h}^{t}} \int_{\partial T}\left|\left(\boldsymbol{u}_{h}-\boldsymbol{\omega}\right) \cdot \boldsymbol{n}\right|\left|\operatorname{tang}\left(\boldsymbol{u}_{h}-\widehat{\boldsymbol{u}}_{h}\right)\right|^{2} \mathrm{ds} .
$$

Proof. Taking the test functions $\left(\boldsymbol{v}_{h}, q_{h}, \widehat{\boldsymbol{v}}_{h}, \widehat{\tau}_{h}^{n n}\right):=\left(\boldsymbol{u}_{h}, p_{h}, \widehat{\boldsymbol{u}}_{h}, \widehat{\sigma}_{h}^{n n}\right)$ and adding, we get

$$
\rho \mathcal{M}_{h}\left(\left.\frac{\partial \boldsymbol{u}_{h}}{\partial t}\right|_{\mathbf{x}_{0}}, \boldsymbol{u}_{h}\right)+\rho \mathcal{C}_{h}\left(\left(\boldsymbol{\omega}, \boldsymbol{u}_{h}, \widehat{\boldsymbol{u}}_{h}\right),\left(\boldsymbol{u}_{h}, \widehat{\boldsymbol{u}}_{h}\right)\right)+2 \mu \mathcal{B}_{h}\left(\left(\boldsymbol{u}_{h}, \widehat{\boldsymbol{u}}_{h}\right),\left(\boldsymbol{u}_{h}, \widehat{\boldsymbol{u}}_{h}\right)\right)=f_{h}\left(\boldsymbol{v}_{h}\right) .
$$

Using the Reynolds transport theorem, we have

$$
\mathcal{M}_{h}\left(\left.\frac{\partial \boldsymbol{u}_{h}}{\partial t}\right|_{\mathbf{x}_{0}}, \boldsymbol{u}_{h}\right)=\frac{1}{2} \frac{d}{d t}\left\|\boldsymbol{u}_{h}\right\|_{L^{2}\left(\Omega^{t}\right)}^{2}-\frac{1}{2} \int_{\Omega^{t}}\left|\boldsymbol{u}_{h}\right|^{2} \operatorname{div}_{\mathbf{x}} \boldsymbol{\omega} \mathrm{dx} .
$$

By equations (7b), we have $\boldsymbol{u}_{h}$ is divergence-conforming and divergence-free. Hence, the nonlinear term $\mathcal{C}_{h}\left(\left(\boldsymbol{\omega}, \boldsymbol{u}_{h}, \widehat{\boldsymbol{u}}_{h}\right),\left(\boldsymbol{u}_{h}, \widehat{\boldsymbol{u}}_{h}\right)\right)$ can be simplified as

$$
\frac{1}{2} \sum_{T \in \mathcal{T}_{h}^{t}} \int_{\partial T}\left|\left(\boldsymbol{u}_{h}-\boldsymbol{\omega}\right) \cdot \boldsymbol{n}\right|\left|\operatorname{tang}\left(\boldsymbol{u}_{h}-\widehat{\boldsymbol{u}}_{h}\right)\right|^{2} \mathrm{ds}+\frac{1}{2} \int_{\Omega^{t}}\left|\boldsymbol{u}_{h}\right|^{2} \operatorname{div}_{\mathbf{x}} \boldsymbol{\omega} \mathrm{dx} .
$$

Combining the above two expressions, we arrive at the desired energy identity in Theorem 2.2 . 
2.3.2. Fully implicit scheme. Let $\left\{\boldsymbol{\phi}_{i}^{t}:=\phi_{i}^{0} \circ \mathcal{A}_{t}^{-1}\right\}_{i=1}^{N}$ be a set of basis functions for $\boldsymbol{V}_{h}^{t}$ where $\left\{\boldsymbol{\phi}_{i}^{0}\right\}_{i=1}^{N}$ is a set of basis functions for $\boldsymbol{V}_{h}^{0}$. We observe that the following time derivative of the basis functions vanishes:

$$
\left.\frac{\partial \phi_{i}^{t}}{\partial t}\right|_{\mathbf{x}_{0}}=\left.\frac{\partial \phi_{i}^{0}}{\partial t}\right|_{\mathbf{x}_{0}} \circ \mathcal{A}_{t}^{-1}=0
$$

Now, denote $\left\{t^{n}:=n \delta t\right\}_{n=0}^{M}$ be the uniform partition of the time interval $[0, T]$, with time step $\delta t$ and $T=M \delta t$. Let

$$
\boldsymbol{u}_{h}^{m}:=\sum_{i=1}^{N} \boldsymbol{\phi}_{i}^{t^{m}}(\mathbf{x}) u_{i}^{m} \in \boldsymbol{V}_{h}^{t^{m}}
$$

be the velocity approximation at time $t^{m}$. The pull-back of $\boldsymbol{u}_{h}^{m}$ to any other configuration $\Omega_{s}$ with is given by

$$
\boldsymbol{u}_{h}^{m} \circ \mathcal{A}_{s} \circ\left(\mathcal{A}_{t^{m}}\right)^{-1}=\sum_{i=1}^{N} \boldsymbol{\phi}_{i}^{s}(\mathbf{x}) u_{i}^{m} \in \boldsymbol{V}_{h}^{s}
$$

which, to lighten notation, we still denote as $\boldsymbol{u}_{h}^{m}$. We use backward difference formula (BDF) to discretize the time derivative term in (7a). So we introduce the backward discretization operators (up to third order accuracy) applied to the function $\boldsymbol{u}_{h}^{m}$ :

$$
\begin{array}{rlrl}
D_{t}^{1} \boldsymbol{u}_{h}^{m} & :=\frac{1}{\delta t}\left(\boldsymbol{u}_{h}^{m}-\boldsymbol{u}_{h}^{m-1}\right), & & \text { BDF1, } \\
D_{t}^{2} \boldsymbol{u}_{h}^{m}:=\frac{1}{\delta t}\left(\frac{3}{2} \boldsymbol{u}_{h}^{m}-2 \boldsymbol{u}_{h}^{m-1}+\frac{1}{2} \boldsymbol{u}_{h}^{m-2}\right), & & \text { BDF2, } \\
D_{t}^{3} \boldsymbol{u}_{h}^{m}:=\frac{1}{\delta t}\left(\frac{11}{6} \boldsymbol{u}_{h}^{m}-3 \boldsymbol{u}_{h}^{m-1}+\frac{3}{2} \boldsymbol{u}_{h}^{m-2}-\frac{1}{3} \boldsymbol{u}_{h}^{m-3}\right), & & \text { BDF3. }
\end{array}
$$

Finally, the fully discrete scheme with BDF time discretization reads as follows: Given $s \in\{1,2,3\}$ and $\boldsymbol{u}_{h}^{j}$ for $j \leq s-1$, find, for $m \in\{s, \cdots, M\},\left(\boldsymbol{u}_{h}^{m}, p_{h}^{m}, \widehat{\boldsymbol{u}}_{h}^{m}, \widehat{\sigma}_{h}^{n n, m}\right) \in \boldsymbol{V}_{h}^{t_{m}} \times Q_{h}^{t_{m}} \times \widehat{\boldsymbol{V}}_{h, 0}^{t_{m}} \times \widehat{M}_{h}^{t_{m}}$ such that

$$
\begin{aligned}
\rho \mathcal{M}_{h}\left(D_{t}^{s} \boldsymbol{u}_{h}^{m}, \boldsymbol{v}_{h}\right)+\rho \mathcal{C}_{h}\left(\left(\boldsymbol{\omega}, \boldsymbol{u}_{h}^{m}, \widehat{\boldsymbol{u}}_{h}^{m}\right),\left(\boldsymbol{v}_{h}, \widehat{\boldsymbol{v}}_{h}\right)\right) & \\
+2 \mu \mathcal{B}_{h}\left(\left(\boldsymbol{u}_{h}^{m}, \widehat{\boldsymbol{u}}_{h}^{m}\right),\left(\boldsymbol{v}_{h}, \widehat{\boldsymbol{v}}_{h}\right)\right)-\mathcal{D}_{h}\left(\boldsymbol{v}_{h},\left(p_{h}^{m}, \widehat{\sigma}_{h}^{n n, m}\right)\right) & =f_{h}\left(\boldsymbol{v}_{h}\right), \\
\mathcal{D}_{h}\left(\boldsymbol{u}_{h}^{m},\left(q_{h}, \widehat{\tau}_{h}^{n n}\right)\right) & =0,
\end{aligned}
$$

for all $\left(\boldsymbol{v}_{h}, q_{h}, \widehat{\boldsymbol{v}}_{h}, \widehat{\tau}_{h}^{n n}\right) \in \boldsymbol{V}_{h}^{t_{m}} \times Q_{h}^{t_{m}} \times \widehat{\boldsymbol{V}}_{h, 0}^{t_{m}} \times \widehat{M}_{h}^{t_{m}}$. We note that the fully discrete scheme results in a system of nonlinear equations due to the implicit treatment of the nonlinear convection term. It has been shown in [40] that a fully discrete scheme is only conditionally stable even if it is based on BDF1 time discretization, with maximum allowable time step depends on the speed of domain deformation. Hence, it makes sense to treat the (nonlinear) convection term in (2) explicitly to yield a less computational intensive, conditionally stable linear scheme as we show next.

2.3.3. Implicit-Explicit scheme. Here we treat the nonlinear convection term explicitly with a standard upwinding DG formulation:

$$
\mathcal{C}_{h}^{d g}\left(\boldsymbol{w}, \boldsymbol{u}_{h}, \boldsymbol{v}_{h}\right):=\sum_{T \in \mathcal{T}_{h}^{t}}-\int_{T} \boldsymbol{u}_{h} \cdot \operatorname{div}_{\mathbf{x}}\left(\left(\boldsymbol{u}_{h}-\boldsymbol{\omega}\right) \otimes \boldsymbol{v}_{h}\right) \mathrm{dx}+\int_{\partial T}\left(\boldsymbol{u}_{h}-\boldsymbol{\omega}\right) \cdot \boldsymbol{n} \operatorname{tang}\left(\boldsymbol{u}_{h}^{-}\right) \cdot \operatorname{tang}\left(\boldsymbol{v}_{h}\right) \mathrm{ds},
$$

where $\left.\boldsymbol{u}_{h}^{-}\right|_{F}=\left.\left(\left.\boldsymbol{u}_{h}\right|_{T^{-}}\right)\right|_{F}$ is the upwinding numerical flux on the facet $F$ with $T^{-}$the element sharing the facet $F$ such that $\left.\left(\boldsymbol{u}_{h}-\boldsymbol{\omega}\right) \cdot \boldsymbol{n}_{T^{-}}\right|_{F}>0$. The implicit-explicit (IMEX) scheme with 
IMEX-SBDF 2 temporal discretization reads as follows: Given $s \in\{1,2,3\}$ and $\boldsymbol{u}_{h}^{j}$ for $j \leq s-1$, find, for $m \in\{s, \cdots, M\},\left(\boldsymbol{u}_{h}^{m}, p_{h}^{m}, \widehat{\boldsymbol{u}}_{h}^{m}, \widehat{\sigma}_{h}^{n n, m}\right) \in \boldsymbol{V}_{h}^{t_{m}} \times Q_{h}^{t_{m}} \times \widehat{\boldsymbol{V}}_{h, 0}^{t_{m}} \times \widehat{M}_{h}^{t_{m}}$ such that

$$
\begin{aligned}
\rho \mathcal{M}_{h}\left(D_{t}^{s} \boldsymbol{u}_{h}^{m}, \boldsymbol{v}_{h}\right)+\rho \mathcal{C}_{h}^{d g}\left(\boldsymbol{\omega}, \widetilde{\boldsymbol{u}}_{h}^{m, s}, \boldsymbol{v}_{h}\right) & \\
+2 \mu \mathcal{B}_{h}\left(\left(\boldsymbol{u}_{h}^{m}, \widehat{\boldsymbol{u}}_{h}^{m}\right),\left(\boldsymbol{v}_{h}, \widehat{\boldsymbol{v}}_{h}\right)\right)-\mathcal{D}_{h}\left(\boldsymbol{v}_{h},\left(p_{h}^{m}, \widehat{\sigma}_{h}^{n n, m}\right)\right) & =f_{h}\left(\boldsymbol{v}_{h}\right), \\
\mathcal{D}_{h}\left(\boldsymbol{u}_{h}^{m},\left(q_{h}, \widehat{\tau}_{h}^{n n}\right)\right) & =0
\end{aligned}
$$

for all $\left(\boldsymbol{v}_{h}, q_{h}, \widehat{\boldsymbol{v}}_{h}, \widehat{\tau}_{h}^{n n}\right) \in \boldsymbol{V}_{h}^{t_{m}} \times Q_{h}^{t_{m}} \times \widehat{\boldsymbol{V}}_{h, 0}^{t_{m}} \times \widehat{M}_{h}^{t_{m}}$, where

$$
\widetilde{\boldsymbol{u}}_{h}^{m, s}:= \begin{cases}\boldsymbol{u}_{h}^{m-1}, & \text { for } s=1, \\ 2 \boldsymbol{u}_{h}^{m-1}-\boldsymbol{u}_{h}^{m-2}, & \text { for } s=2, \\ 3 \boldsymbol{u}_{h}^{m-1}-3 \boldsymbol{u}_{h}^{m-2}+\boldsymbol{u}_{h}^{m-3}, & \text { for } s=3 .\end{cases}
$$

The IMEX scheme (9) is linear and the resulting global linear system after static condensation is a symmetric Stokes-like system with global unknowns consists of tangential velocity and normalnormal stress on the mesh skeleton.

Remark 2.3. When the flow problem (2) is convection-dominated and the mesh is under-resolved for the viscous term (which is typical the case for high Reynolds number flows), it further make sense to treat the second-order viscous term in (2) explicitly with a DG formulation [9] leaving only the velocity-pressure coupling implicit, see our recent studies [17, 18] on static meshes.

\section{The ALE-TVNNS-HDG SCHEME FOR TWO-PHASE FLOW}

In this section, we extend the ALE-TVNNS-HDG scheme proposed in Section 2 to incompressible two-phase flow with surface tension. Assume a geometrical setting as sketched in Fig. 1. denote by

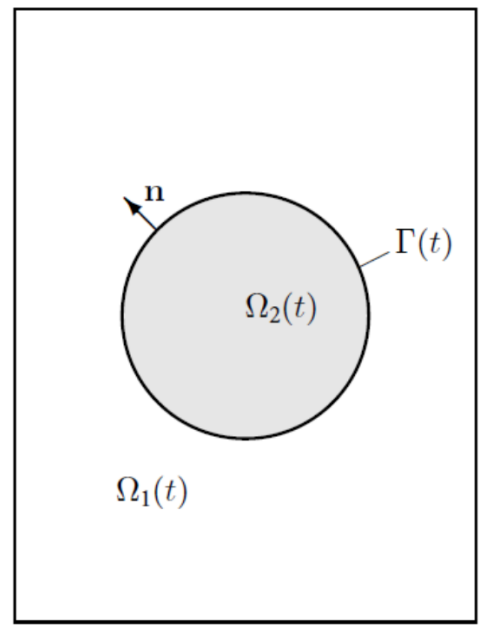

FiguRE 1. Schematic view of the two-phase domain.

$\Omega$ a fixed domain such that $\bar{\Omega}=\bar{\Omega}_{1}(t) \cup \bar{\Omega}_{2}(t)$, where $\Omega_{1}(t), \Omega_{2}(t)$ denote two domains separated by a sharp smooth interface $\Gamma(t):=\bar{\Omega}_{1}(t) \cap \bar{\Omega}_{2}(t)$ with normal vector $\boldsymbol{n}$ pointing from $\Omega_{2}(t)$ into $\Omega_{1}(t)$. Assume the domain $\Omega_{i}$ occupies an incompressible fluid with densitiy $\rho_{i}$ and dynamic viscosity $\mu_{i}$ for $i=1,2$. Then, the immiscible incompressible two-phase flow equations with surface tension reads as follows: Given an initial interface $\Gamma_{0} \subset \Omega$ and corresponding sub-domains $\Omega_{i}(0)$, initial 
velocity $\boldsymbol{u}_{0}$ and volume force $\boldsymbol{f}$, find a velocity $\boldsymbol{u}(\mathbf{x}, t)$, pressure $p(\mathbf{x}, t)$, and interface $\Gamma(t)$ such that $\boldsymbol{u}(\cdot, 0)=\boldsymbol{u}_{0}, \Gamma(0)=\Gamma_{0}$, and for all $t \in[0, T]$ the following equations hold,

$$
\begin{array}{rlrl}
\rho_{i}\left(\frac{\partial \boldsymbol{u}}{\partial t}+\boldsymbol{u} \cdot \nabla_{\mathbf{x}} \boldsymbol{u}\right)-\operatorname{div}_{\mathbf{x}} \boldsymbol{\sigma}_{i}=\rho_{i} \boldsymbol{f}, & & \text { in } \Omega_{i}, & i=1,2 \\
\operatorname{div}_{\mathbf{x}} \boldsymbol{u}=0, & \text { in } \Omega_{i}, \quad i=1,2, \\
\boldsymbol{u}=0, & \text { on } \partial \Omega, \\
{[\boldsymbol{\sigma n}]=-\tau \kappa \boldsymbol{n}, \quad[\boldsymbol{u}]=0,} & \text { on } \Gamma, \\
V_{\Gamma}=\boldsymbol{u} \cdot \boldsymbol{n}, & \text { on } \Gamma,
\end{array}
$$

where $\boldsymbol{\sigma}_{i}:=2 \mu_{i} \mathbf{D}_{\mathbf{x}}(\boldsymbol{u})-p \boldsymbol{I}$ is the stress on domain $\Omega_{i}, \tau$ is the surface tension coefficient assumed to be constant, the scalar function

$$
\kappa(\mathbf{x}):=\operatorname{div}_{\mathbf{x}} \boldsymbol{n}(\mathbf{x}), \quad \mathbf{x} \in \Gamma
$$

is the mean curvature, and $V_{\Gamma}=V_{\Gamma}(\mathbf{x}, t) \in \mathbb{R}$ denotes the size of the velocity of the interface $\Gamma$ at $\mathbf{x} \in \Gamma(t)$.

We solve the moving interface problem (10) on ALE moving interface-fitted meshes. In particular, we combine the IMEX-ALE-TVNNS-HDG scheme (9) with a semi-implicit treatment 14 of the domain motion where the interface is updated explicitly, which results in a linear scheme. We use the Laplace-Beltrami technique to approximate the mean curvature vector $\kappa \boldsymbol{n}$ on the discrete interface. Details of the fully discrete algorithm using second-order IMEX-SBDF time stepping $(s=2)$ is given below.

We start with an interface-fitted conforming simplicial mesh $\mathcal{T}_{h}^{0}=\mathcal{T}_{h}^{1,0} \cup \mathcal{T}_{h}^{2,0}$ of the domain $\Omega$ where $\mathcal{T}_{h}^{i, 0}$ is a triangulation of $\Omega_{i}(0)$ for $i=1,2$, and $\Gamma_{h}^{0}:=\overline{\mathcal{T}_{h}^{1,0}} \cap \overline{\mathcal{T}_{h}^{2,0}}$ is a discretization of the interface $\Gamma_{0}$. Again, we denote $\left\{t^{n}:=n \delta t\right\}_{n=0}^{M}$ be the uniform partition of the time interval $[0, T]$ with time step $\delta t$ and $T=M \delta t$. Given $\boldsymbol{u}_{h}^{j} \in \boldsymbol{V}_{h}^{j}$ as the velocity approximation at time $t^{j}$ with $j=0,1$, and let $\mathcal{T}_{h}^{1}:=\mathcal{A}_{h}^{1}\left(\mathcal{T}_{h}^{0}\right)$ be the mapped mesh at time $t^{1}$ with $\Gamma_{h}^{1}:=\mathcal{A}_{h}^{1}\left(\Gamma_{h}^{0}\right)$ the interface approximation at $t^{1}$. Then, for $m \in\{2, \cdots, M\}$ we perform the following four steps to update the computational mesh and field unknows at time $t^{m}$ :

- Step 1: Extrapolate the interface $\Gamma_{h}^{m}$ with a second-order Adams-Bashforth method:

$$
\Gamma_{h}^{m}\left(\mathbf{x}^{m-1}\right)=\mathbf{x}^{m-1}+\delta t \boldsymbol{\omega}_{\Gamma}^{m-1 / 2}, \quad \forall \mathbf{x}^{m-1} \in \Gamma_{h}^{m-1},
$$

where $\omega_{\Gamma}^{m-1 / 2}$ is the mesh velocity on the interface that satisfies

$$
\left.\boldsymbol{\omega}_{\Gamma}^{m-1 / 2} \cdot \boldsymbol{n}\right|_{\Gamma_{h}^{m-1}}=\left.\left(1.5 \boldsymbol{u}^{m-1}-0.5 \boldsymbol{u}^{m-2}\right) \cdot \boldsymbol{n}\right|_{\Gamma_{h}^{m-1}} .
$$

Note that the tangential component of mesh velocity $\omega_{\Gamma}^{m-1 / 2}$ can be freely chosen as it does not affect the shape of the interface.

- Step 2: Define the new mesh $\mathcal{T}_{h}^{m}=\mathcal{A}_{h}^{m}\left(\mathcal{T}_{h}^{m-1}\right)$ by an extension operator:

$$
\mathcal{A}_{h}^{m}\left(\mathbf{x}^{m-1}\right)=\mathbf{x}^{m-1}+\operatorname{Ext}\left(\delta t \omega_{\Gamma}^{m-1 / 2}\right),
$$

where Ext is a proper extension operator from the interface to the interior domain.

- Step 3: Update the computation mesh $\mathcal{T}_{h}^{m}$ and the interface $\Gamma_{h}^{m}$ and compute the approximatio to the mean curvature vector $\left.\kappa \boldsymbol{n}\right|_{\Gamma_{h}^{m}}=-\left.\triangle_{\Gamma}\left(\mathbf{x}^{m}\right)\right|_{\Gamma_{h}^{m}}$ by the Laplace-Beltrami technique: Find $\boldsymbol{\kappa}^{m} \in\left[V_{\Gamma_{h}^{m}}^{k}\right]^{d}$ such that

$$
\left(\boldsymbol{\kappa}^{m}, \boldsymbol{\psi}\right)_{\Gamma_{h}^{m}}=\left.\left(\nabla_{\Gamma}\left(\mathbf{x}^{m}\right), \nabla_{\Gamma} \boldsymbol{\psi}\right)\right|_{\Gamma_{h}^{m}}, \quad \forall \boldsymbol{\psi} \in\left[\mathcal{V}_{\Gamma_{h}^{m}}^{k}\right]^{d},
$$

where

$$
\mathcal{V}_{\Gamma_{h}^{m}}^{k}:=\left\{\psi \in H^{1}\left(\Gamma_{h}^{m}\right):\left.\quad \psi\right|_{F} \in \mathcal{P}^{k}(F), \quad \forall F \in \Gamma_{h}^{m}\right\}
$$


Here $\nabla_{\Gamma}(\bullet):=\nabla(\bullet)-(\nabla(\bullet) \cdot \boldsymbol{n}) \boldsymbol{n}$ is the surface gradient, and $\triangle_{\Gamma}$ is the surface Laplacian. We also compute the mesh velocity $\boldsymbol{\omega}^{m}$ at time $t^{m}$ by BDF2:

$$
\boldsymbol{\omega}^{m}:=\frac{1}{\delta t}\left(1.5 \mathcal{A}_{h}^{m}-2 \mathcal{A}_{h}^{m-1}+0.5 \mathcal{A}_{h}^{m-2}\right) .
$$

- Step 4: Update field unknowns at $t^{m}$ by the IMEX-ALE-TVNNS-HDG scheme (9) (with $\overline{s=2)}$, where the density $\rho$ and viscosity $\mu$ in equation (9a) are understood to be piecewise constant functions that need to be placed inside the operators, and the right hand side of (9a) has the following additional term due to surface tension:

$$
f_{\Gamma_{h}}\left(\boldsymbol{\kappa}_{h}^{m}, \boldsymbol{v}_{h}\right):=\sum_{F \in \Gamma_{h}^{m}} \int_{F} \tau \boldsymbol{\kappa}_{h}^{m} \cdot \boldsymbol{v}_{h}^{-} \mathrm{ds},
$$

where $\left.\boldsymbol{v}_{h}^{-}\right|_{F}=\left.\left(\left.\left(\boldsymbol{v}_{h}\right)\right|_{K^{-}}\right)\right|_{F}$ with $K^{-}$being the element sharing the facet $F$ that belongs to the interior subdomain $\Omega_{2}$. For completeness, we present the resulting fully discrete formulation below: Find $\left(\boldsymbol{u}_{h}^{m}, p_{h}^{m}, \widehat{\boldsymbol{u}}_{h}^{m}, \widehat{\sigma}_{h}^{n n, m}\right) \in \boldsymbol{V}_{h}^{t_{m}} \times Q_{h}^{t_{m}} \times \widehat{\boldsymbol{V}}_{h, 0}^{t_{m}} \times \widehat{M}_{h}^{t_{m}}$ such that

$$
\begin{aligned}
\mathcal{M}_{h}\left(D_{t}^{2} \boldsymbol{u}_{h}^{m}, \rho \boldsymbol{v}_{h}\right)+\mathcal{C}_{h}^{d g}\left(\boldsymbol{\omega}^{m}, \widetilde{\boldsymbol{u}}_{h}^{m, 2}, \rho \boldsymbol{v}_{h}\right) & \\
+\mathcal{B}_{h}\left(\left(\boldsymbol{u}_{h}^{m}, \widehat{\boldsymbol{u}}_{h}^{m}\right),\left(2 \mu \boldsymbol{v}_{h}, 2 \mu \widehat{\boldsymbol{v}}_{h}\right)\right)-\mathcal{D}_{h}\left(\boldsymbol{v}_{h},\left(p_{h}^{m}, \widehat{\sigma}_{h}^{n n, m}\right)\right) & =f_{h}\left(\boldsymbol{v}_{h}\right)+f_{\Gamma_{h}}\left(\boldsymbol{\kappa}_{h}^{m}, \boldsymbol{v}_{h}\right), \\
\mathcal{D}_{h}\left(\boldsymbol{u}_{h}^{m},\left(q_{h}, \widehat{\tau}_{h}^{n n}\right)\right) & =0,
\end{aligned}
$$

for all $\left(\boldsymbol{v}_{h}, q_{h}, \widehat{\boldsymbol{v}}_{h}, \widehat{\tau}_{h}^{n n}\right) \in \boldsymbol{V}_{h}^{t_{m}} \times Q_{h}^{t_{m}} \times \widehat{\boldsymbol{V}}_{h, 0}^{t_{m}} \times \widehat{M}_{h}^{t_{m}}$.

Remark 3.1 (Step 1: Interface node distribution). The choice of $\omega_{\Gamma}^{m-1 / 2}$ strongly affect the node distributions on the interface. Traditionally, $\omega_{\Gamma}^{m-1 / 2}$ is chosen to be, either the full fluid velocity

$$
\boldsymbol{\omega}_{\Gamma}^{m-1 / 2}=1.5 \boldsymbol{u}^{m-1}-0.5 \boldsymbol{u}^{m-2},
$$

or the normal component of the fluid velocity

$$
\boldsymbol{\omega}_{\Gamma}^{m-1 / 2}=\left(\left(1.5 \boldsymbol{u}^{m-1}-0.5 \boldsymbol{u}^{m-2}\right) \cdot \boldsymbol{n}\right) \boldsymbol{n} .
$$

However, both approaches suffer from the general problem that there is no control of the node distribution and of the shape of surface elements. We follow the work [3], see also [20], to update the mesh velocity that combines the equation (11) with the Laplace-Beltrami identity $-\triangle_{\Gamma} \mathrm{id}=\kappa \boldsymbol{n}$ for the curvature in order to get more control over the node distribution. In particular, we find $\left(\boldsymbol{\omega}_{\Gamma}^{m-1 / 2}, \kappa_{h}\right) \in\left[\mathcal{V}_{\Gamma_{h}^{m-1}}^{k}\right]^{d} \times \mathcal{V}_{\Gamma_{h}^{m-1}}^{k}$ such that

$$
\begin{aligned}
\left\langle\boldsymbol{\omega}_{\Gamma}^{m-1 / 2} \cdot \boldsymbol{n}, \phi\right\rangle_{\Gamma_{h}^{m-1}} & =\left\langle\left(1.5 \boldsymbol{u}^{m-1}-0.5 \boldsymbol{u}^{m-2}\right) \cdot \boldsymbol{n}, \phi\right\rangle_{\Gamma_{h}^{m-1}}, \\
\langle\kappa, \boldsymbol{\psi} \cdot \boldsymbol{n}\rangle_{\Gamma_{h}^{m-1}}-\delta t\left\langle\nabla_{\Gamma} \boldsymbol{\omega}_{\Gamma}^{m-1 / 2}, \nabla_{\Gamma} \boldsymbol{\psi}\right\rangle_{\Gamma_{h}^{m-1}} & =\left\langle\nabla_{\Gamma} \mathbf{x}^{m-1}, \nabla_{\Gamma} \boldsymbol{\psi}\right\rangle_{\Gamma_{h}^{m-1}},
\end{aligned}
$$

for all $(\boldsymbol{\psi}, \phi) \in\left[\mathcal{V}_{\Gamma_{h}^{m-1}}^{k}\right]^{d} \times \mathcal{V}_{\Gamma_{h}^{m-1}}^{k}$. Numerical comparion of the approaches (15) and (16) will be presented in Section 4 for the rising bubble benchmark problem [29], which show the superior performance of approach (16) in terms of interface node distribution.

Remark 3.2 (Step 2: Construction of the ALE map). Various procedures were introduced in the literature to extend interface deformation to the interior domain, see, e.g., [4, 16, 56, 57]. In this paper, we construct the $A L E$ map $\mathcal{A}_{h}^{m} \in\left[\mathcal{V}_{h}^{m-1, k}\right]^{d}$ using harmonic extension with stiffening [45] on the updated mesh $\mathcal{T}_{h}^{m-1}$ as follows: Find $\mathcal{A}_{h}^{m} \in\left[\mathcal{V}_{h}^{m-1, k}\right]^{d}$ with $\left.\mathcal{A}_{h}^{m}\right|_{\Gamma_{h}^{m-1}}=\mathbf{x}^{m-1}+\delta$ t $\boldsymbol{\omega}_{\Gamma}^{m-1 / 2}$ such that

$$
\left(\alpha \nabla \mathcal{A}_{h}^{m}, \nabla \phi\right)_{\mathcal{T}_{h}^{m-1}}=0, \quad \forall \phi \in\left[\mathcal{V}_{h}^{m-1, k}\right]^{d} \text { with }\left.\phi\right|_{\Gamma_{h}^{m-1}}=0,
$$


where the stiffening coefficient $\alpha: \mathcal{T}_{h}^{m-1} \rightarrow \mathbb{R}$ takes maximum value 10 on the interface and decreases rapidly to 1 away from the interface based on the distance of the point to the nearest interface point, and the space

$$
\mathcal{V}_{h}^{m-1, k}:=\left\{\psi \in H_{0}^{1}\left(\mathcal{T}_{h}^{m-1}\right):\left.\quad \psi\right|_{T} \in \mathcal{P}^{k}(T), \quad \forall T \in \mathcal{T}_{h}^{m-1}\right\} .
$$

Remark 3.3 (Step 3: Mean curvature vector approximation). We use an $L^{2}$-project (12) with isoparametric finite elements of degree $k$ to approximate the mean curvature vector. It was shown in [24] that the order of convergence in the $L^{2}$-norm for the approximation is only $k-1$. This will negatively impact the accuracy of the velocity approximation in Step 4, which in turn negatively affects the accuracy of the interface approximation in Step 1. The construction of more accurate mean curvature vector approximation consists of our ongoing work. We mention that mean curvature vector approximation with first order $L^{2}$-convergence can be obtained for linear finite elements with stabilization on piecewise linear surface [23].

Remark 3.4 (Step 4: Consistency). We show that the equation (14a) is a consistent discretization of the momentum equation (10a) with the interface condition (10d). Let $\boldsymbol{u}(\mathbf{x}, t)$ and $p(\mathbf{x}, t)$ be the smooth solution to the equations (10). For simplicity, we only consider the semi-discrete case (continuous in time). Assume

$$
\left.\widehat{\sigma}^{n n}\right|_{F}:= \begin{cases}(\boldsymbol{\sigma n}) \cdot \boldsymbol{n} & \text { if } F \notin \Gamma_{h}^{m}, \\ \left(\boldsymbol{\sigma}_{1} \boldsymbol{n}\right) \cdot \boldsymbol{n} & \text { if } F \in \Gamma_{h}^{m},\end{cases}
$$

Then, by intergration by parts, we have

$$
\begin{aligned}
& \mathcal{M}_{h}\left(\left.\frac{\partial \boldsymbol{u}}{\partial t}\right|_{\mathbf{x}_{0}}, \rho \boldsymbol{v}_{h}\right)+\mathcal{C}_{h}^{d g}\left(\boldsymbol{\omega}, \boldsymbol{u}, \rho \boldsymbol{v}_{h}\right)+\mathcal{B}_{h}\left((\boldsymbol{u}, \boldsymbol{u}),\left(2 \mu \boldsymbol{v}_{h}, 2 \mu \widehat{\boldsymbol{v}}_{h}\right)\right)-\mathcal{D}_{h}\left(\boldsymbol{v}_{h},\left(p, \widehat{\sigma}^{n n}\right)\right) \\
& =\left(\rho\left(\left.\frac{\partial \boldsymbol{u}}{\partial t}\right|_{\mathbf{x}_{0}}+(\boldsymbol{u}-\boldsymbol{\omega}) \cdot \nabla \boldsymbol{u}\right)-\operatorname{div}_{\mathbf{x}} \boldsymbol{\sigma}, \boldsymbol{v}_{h}\right)_{\mathcal{T}_{h}^{m}}-\left\langle\left(\boldsymbol{\sigma}_{2}-\boldsymbol{\sigma}_{1}\right) \boldsymbol{n}_{2}, \boldsymbol{v}_{h}^{-}\right\rangle_{\Gamma_{h}^{m}} \\
& =\left(\rho \boldsymbol{f}, \boldsymbol{v}_{h}\right)_{\mathcal{T}_{h}^{m}}+\left\langle\tau \kappa \boldsymbol{n}_{2}, \boldsymbol{v}_{h}^{-}\right\rangle_{\Gamma_{h}^{m}} \\
& =f_{h}\left(\boldsymbol{v}_{h}\right)+f_{\Gamma_{h}}\left(\kappa \boldsymbol{n}_{2}, \boldsymbol{v}_{h}\right),
\end{aligned}
$$

where $\boldsymbol{n}_{2}$ is the normal vector pointing from $\Omega_{2}$ to $\Omega_{1}$. Hence, the spatial discretization in equation (14a) is consistent with the equations (10a)-(10d).

\section{Numerical Results}

Here we show three numerical studies for the IMEX-ALE-TVNNS-HDG schemes (9) and (14). The first example focus on the accuracy study of the proposed method with a prescribed smooth ALE map. We observe optimal convergence. The second example deals with the application of our method to a free boundary problem, where the ALE map is not a priori given. The last example solves the classical rising bubble benchmark problem [29] in two-phase flow. Our numerical simulations are performed using the open-source finite-element software NGSolve [46, https:// ngsolve.org/.

4.1. Accuracy test. We consider the Navier-Stokes equations $(2)$ on the domain $\Omega^{t}=[0,1] \times[0,1]$ for $t \in[0,0.5 \pi]$ with homogeneous Dirichlet boundary conditions for velocity, and choose the source term such that the exact solution is given as follows:

$$
\left\{\begin{array}{l}
u_{1}(\mathbf{x}, t)=4\left(x_{1}\left(1-x_{1}\right)\right)^{2}\left(2 x_{2}-6 x_{2}^{2}+4 x_{2}^{3}\right) \sin (t), \\
u_{2}(\mathbf{x}, t)=-4\left(x_{2}\left(1-x_{2}\right)\right)^{2}\left(2 x_{1}-6 x_{1}^{2}+4 x_{1}^{3}\right) \sin (t),
\end{array} \quad p(\mathbf{x}, t)=\sin \left(x_{1}+x_{2}\right) .\right.
$$


Density $\rho$ is taken to be 1 and we consider viscosity $\mu$ either to be 1 or $10^{-6}$. We use the following prescribed ALE map $\mathbf{x}\left(\mathbf{x}_{0}, t\right)=\mathcal{A}_{t}\left(x_{0,1}, x_{0,2}\right)=\left(x_{0,1}+u_{1}\left(\mathbf{x}_{0}, 2 t\right), x_{0,2}+u_{2}\left(\mathbf{x}_{0}, 2 t\right)\right)$ for the mesh movement, where $\boldsymbol{u}=\left(u_{1}, u_{2}\right), \mathbf{x}=\left(x_{1}, x_{2}\right)$, and $\mathbf{x}_{0}=\left(x_{0,1}, x_{0,2}\right)$.

We compare our HDG method (9), denoted as HDG, with the ALE method using Scott-Volegius element (on barycentric refined meshes), denoted as SV, and that using high-order Taylor-Hood element, denoted as TH. We use either polynomials of degree $k=2$ or $k=3$ for the velocity approximation. For the time discretization, we use the third order IMEX-SBDF3 method [2]. Time step size is taken to be small enough so that spatial error dominates temporal error. Table 1 lists the history of convergence for the $L^{2}$-error in the velocity approximation for $k=2$ and $k=3$. We observe that the order of convergence for the error for all three methods are optimal $(k+1)$ for the case $\mu=1$. But for the convection-dominated case $\mu=10^{-6}$, the accuracy for HDG clearly outperforms those for SV and TH. In particular, HDG velocity error is not affected by the viscosity coefficient, however, SV looses roughly one order of approximation and TH looses up to two order of approximation for the case $\mu=10^{-6}$.

\begin{tabular}{|c|c|c|c|c|c|c|c|c|c|c|c|c|c|}
\hline & & \multicolumn{4}{|c|}{ HDG } & \multicolumn{4}{c|}{ SV } & \multicolumn{4}{c|}{ TH } \\
\hline & & \multicolumn{2}{|c|}{$\mu=1$} & \multicolumn{2}{c|}{$\mu=10^{-6}$} & \multicolumn{2}{c|}{$\mu=1$} & \multicolumn{2}{c|}{$\mu=10^{-6}$} & \multicolumn{3}{c|}{$\mu=1$} & \multicolumn{2}{c|}{$\mu=10^{-6}$} \\
\hline$k$ & $1 / h$ & Error & Order & Error & Order & Error & Order & Error & Order & Error & Order & Error & Order \\
\hline & 8 & $2.27 \mathrm{e}-04$ & - & $1.45 \mathrm{e}-04$ & - & $4.74 \mathrm{e}-04$ & - & $4.93 \mathrm{e}-04$ & - & $1.71 \mathrm{e}-04$ & - & $7.08 \mathrm{e}-03$ & - \\
2 & 16 & $2.24 \mathrm{e}-05$ & 3.34 & $1.88 \mathrm{e}-05$ & 2.95 & $5.49 \mathrm{e}-05$ & 3.11 & $1.11 \mathrm{e}-04$ & 2.15 & $2.12 \mathrm{e}-05$ & 3.01 & $2.04 \mathrm{e}-03$ & 1.80 \\
& 32 & $2.46 \mathrm{e}-06$ & 3.19 & $2.37 \mathrm{e}-06$ & 2.99 & $6.30 \mathrm{e}-06$ & 3.12 & $2.60 \mathrm{e}-05$ & 2.10 & $2.65 \mathrm{e}-06$ & 3.00 & $5.19 \mathrm{e}-04$ & 1.97 \\
\hline & 8 & $1.39 \mathrm{e}-05$ & - & $1.13 \mathrm{e}-05$ & - & $1.88 \mathrm{e}-05$ & - & $3.02 \mathrm{e}-05$ & - & $1.05 \mathrm{e}-05$ & - & $6.62 \mathrm{e}-04$ & - \\
3 & 16 & $7.68 \mathrm{e}-07$ & 4.17 & $7.23 \mathrm{e}-07$ & 3.96 & $1.06 \mathrm{e}-06$ & 4.15 & $2.78 \mathrm{e}-06$ & 3.44 & $6.41 \mathrm{e}-07$ & 4.04 & $1.76 \mathrm{e}-04$ & 1.91 \\
& 32 & $4.46 \mathrm{e}-08$ & 4.11 & $4.57 \mathrm{e}-08$ & 3.99 & $6.15 \mathrm{e}-08$ & 4.10 & $2.90 \mathrm{e}-07$ & 3.26 & $3.93 \mathrm{e}-08$ & 4.03 & $4.25 \mathrm{e}-05$ & 2.05 \\
\hline
\end{tabular}

TABLE 1. History of convergence of the $L^{2}$-error for the velocity approximation.

4.2. Free boundary problem. Here we apply our HDG method (9) to a free boundary problem [13,43 that models the propagation of a solitary wave. The ALE Navier-Stokes equations (2) is solved on the free-boundary domain shown in Fig. 2. The top boundary $\Gamma(t)$ in Fig. 2 is a free

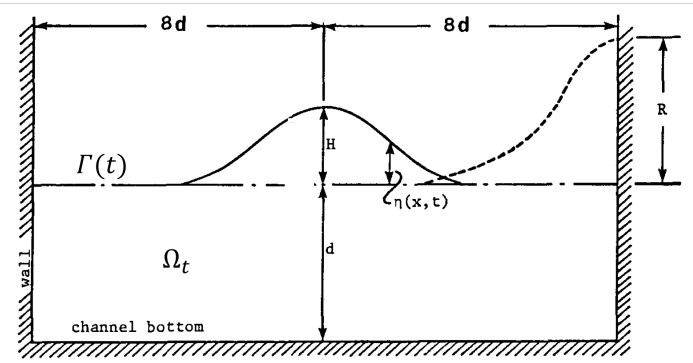

Figure 2. The free-boundary domain.

surface on which we impose a stress-free boundary condition. Free-slip boundary condition is used for the other boundaries. The domain velocity on the free surface is set to be equal to the fluid velocity,

$$
\left.\frac{\partial \mathbf{x}}{\partial t}\right|_{\mathbf{x}_{0}}=\boldsymbol{\omega}(\mathbf{x}, t)=\boldsymbol{u}(\mathbf{x}, t), \quad \forall \mathbf{x} \in \Gamma(t),
$$

which expresses the fact that the free boundary $\Gamma(t)$ consists of the same particles for all $t>0$. The initial condition is taken from Laitone's solitary wave approximation [32], with the free surface elevation $\eta$ given by $\eta=d+H \operatorname{sech}^{2}\left(\sqrt{\frac{3 H}{4 d^{3}}} \mathbf{x}_{1}\right)$, and $H=2$ is the initial wave height and $d=10$ is 
the still water depth. Density and viscosity are taken to be 1, and the source $\boldsymbol{f}$ is a gravitational acceleration of magnitude 9.8 acts vertically on the downward direction.

For this problem the ALE map is not prescribed. We apply the semi-implicit approach [14] to update the domain, see Section 3. We use IMEX-SBDF2 time stepping $(s=2)$ with the HDG scheme (9) using quadratic velocity approximation on a mesh with mesh size $h=2$. Time step size is taken to be $\delta t=0.025$, and the final time $T=12$. A simple harmonic extension on the reference configuration is adopted for the ALE map, which is approximated by continuous linear finite elements. Fig. 3 presents the velocity magnitude at some subsequent times along with the mesh. Our numerical simulation agrees well with computational results given by 13,43 . See
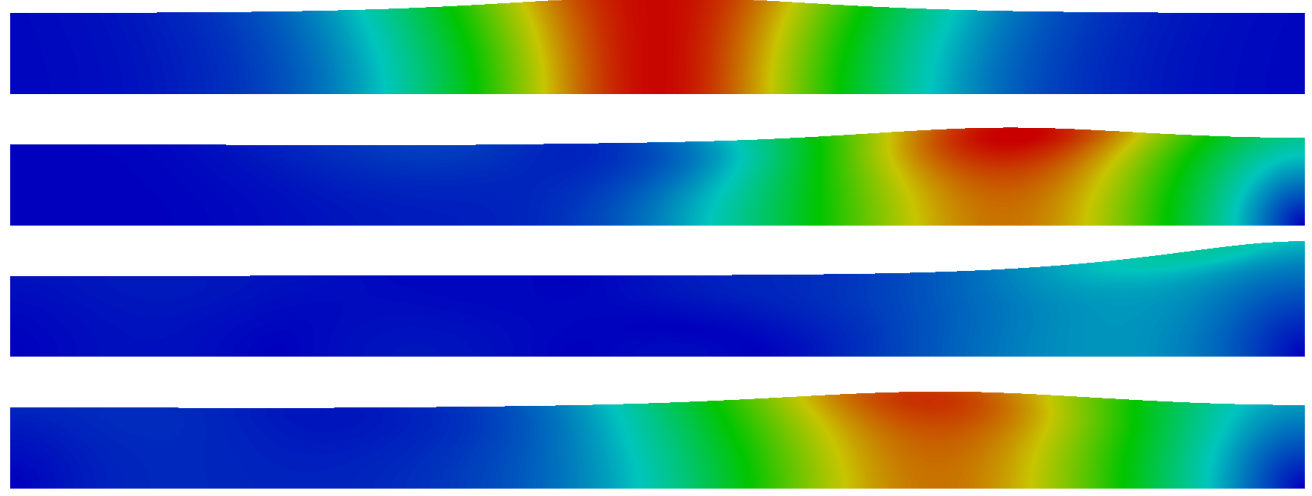

FiguRE 3. Velocity magnitude (from left to right and top to bottom) at time $t=$ $0,4,8$, and 12 .

Table 2 for a comparison of the run-up height, the time when the wave hits the right side wall and the maximum pressure. See also Fig. 4 for the plot of the run-up height versus time, which matches well with numerical results reported in 13 .

\begin{tabular}{|c|c|c|c|}
\hline & $\overline{\text { Duarte }} \overline{13}]$ & Ramaswamy $[\overline{43}]$ & HDG \\
\hline Height & 14.27 & 14.48 & 14.33 \\
\hline Time & 7.7 & 7.6 & 7.65 \\
\hline Pressure & 130 & 131.66 & 131.89 \\
\hline
\end{tabular}

TABLE 2. Comparison table: height, time and pressure.

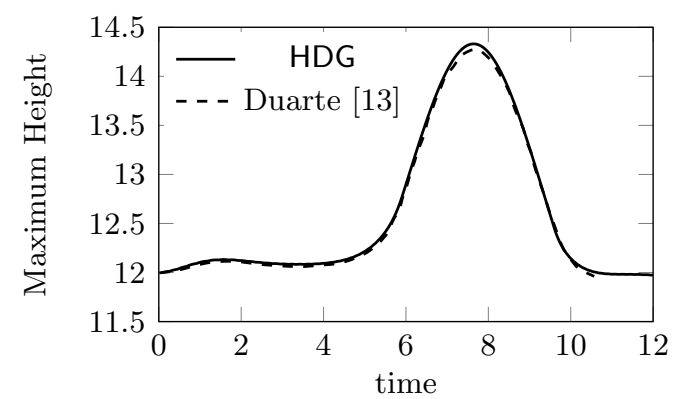

FIGURE 4. Run-up height. 


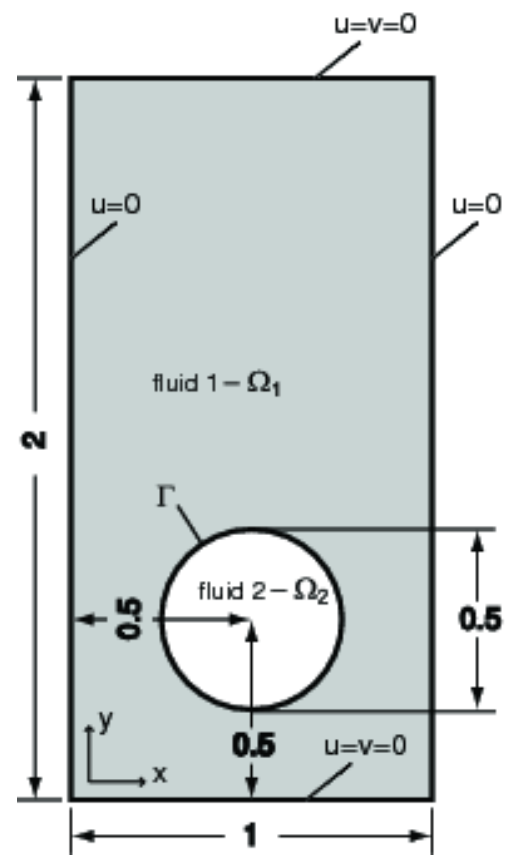

FiguRE 5. Initial configuration and boundary conditions for the rising bubble problem.

4.3. Rising bubble problem. Here we solve the rising bubble problem [29] using the IMEXALE-TVNNS-HDG method detailed in Section 3. The initial configuration, see Fig. 5, consists of a circular bubble of radius $r_{0}=0.25$ centered at $(0.5,0.5)$ in a $[1 \times 2]$ rectangular domain. The density of the bubble is smaller than that of the surrounding fluid $\left(\rho_{2}<\rho_{1}\right)$. The no-slip boundary condition $(\boldsymbol{u}=0)$ is used at the top and bottom boundaries, whereas the free slip condition $(\boldsymbol{u} \cdot \boldsymbol{n}=0,(\boldsymbol{\sigma} \boldsymbol{n}) \cdot \boldsymbol{t}=0)$ is imposed on the vertical walls.

The same two test cases proposed in [29] are considered in this study. Table 3 lists the fluid and physical parameters use for the two test cases. We take final time for Test Case 1 to be $T=3$, and

\begin{tabular}{ccccccc}
\hline Test Case & $\rho_{1}$ & $\rho_{2}$ & $\mu_{1}$ & $\mu_{2}$ & $\boldsymbol{f}$ & $\tau$ \\
1 & 1000 & 100 & 10 & 1 & $(0,-0.98)$ & 24.5 \\
2 & 1000 & 1 & 10 & 0.1 & $(0,-0.98)$ & 1.96 \\
\hline
\end{tabular}

TABLE 3. Physical parameters for the test cases.

for Test Case 2 to be $T=2$. We note that for Test Case 1, the surface tension effects are strong enough to hold the bubble together, which will end up in a ellipsoidal shape, but for Test Case 2 , the decrease in surface tension causes the bubble to assume a more complex shape and develop thin filaments which eventually break off. The time of break up for Test Case 2 is predicted [29] to occur between $t=2.2$ and 2.4. The current ALE scheme without remeshing can not treat interface breakup, so we stop the simulation at time $T=2$ for this case.

For both cases, we consider the IMEX-SBDF2 based ALE-TVNNS-HDG scheme in Section 3 with polynomial degree $k=2$ and quadratic isoparametric triangular elements on three different meshes as depicted in Fig. 6. Mesh M1 has mesh size $h=1 / 20$ away from the interface and $h_{\Gamma}=1 / 20$ near the interface, which consists of 941 nodes and 1260 elements. Mesh M2 has mesh size $h=1 / 20$ away from the interface and $h_{\Gamma}=1 / 40$ near the interface, which consists of 1216 nodes and 2310 elements. Mesh M3 has mesh size $h=1 / 20$ away from the interface and $h_{\Gamma}=1 / 80$ 

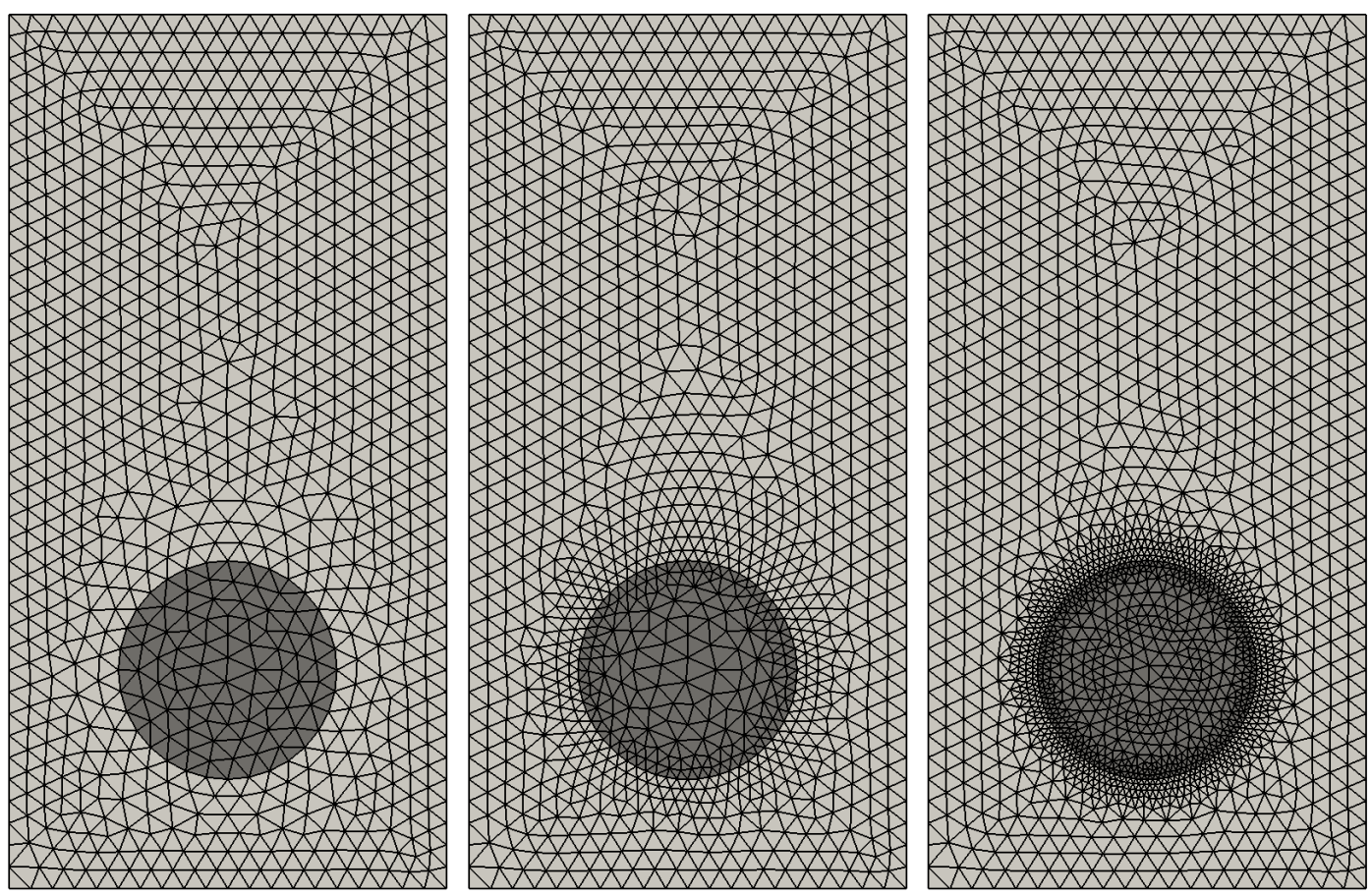

Figure 6. Left: mesh M1. Middle: mesh M2. Right: mesh M3. Dark color: domain $\Omega_{2}$.

near the interface, which consists of 1216 nodes and 2310 elements. For all the simulation, we take time step size to be $\delta t=h_{\Gamma} / 8$ for Test Case 1 and $\delta t=h_{\Gamma} / 4$ for Test Case 2 .

4.3.1. Interface node distribution. We first compare the mesh quality for two different choices of interface velocity updates mentioned in Remark 3.1. We consider Test Case 1 and use the coarse mesh M1. The mesh deformation for the approaches (15) and (16) at time $t=1.5$ and for the approach (16) at time $t=3$ are shown in Fig.7. It is clear to observe that for the approach (15), the interface nodes tends to accumulate at the bottom of the bubble, which eventually leads to mesh tangling and crash of the simulation at around $t=1.65$. However, for the approach (16), the interface nodes seem to be equidistributed, which produce good quality mesh throughout the simulation. For this reason, we choose to use the approach 16 for the interface velocity update for all the tests.

4.3.2. Bubble shape. The bubble shapes at final time are present in Fig. 8. For Test Case 1, we also present the reference data from [29] using the MooNMD code on the finest mesh with 900 degrees of freedom on the interface and 6000 total time steps. We observe that for Test Case 1, the present results are indistinguishable from the reference data even for the coarsest mesh $M 1$. We also observe similar results for Test Case 2 on all three meshes. Zoom-in of the deformed mesh around $\Omega_{2}$ at $t=1$ and $t=2$ for Test Case 2 are shown in Fig. 9. We again observe no interface node clustering for all meshes. However, due to the large deformation of the bubble shape, we observe self-intersection of the mesh for M1 and M2 at time $t=2$ near the left trailing corner of the bubble.

4.3.3. Benchmark quantities. Following [29], we track the following benchmark quantities over time:

(i) Center of mass: the centroid of mass for the bubble

$$
\mathbf{X}_{c}=\left(x_{c}, y_{c}\right)=\frac{\int_{\Omega_{2}} \mathbf{x d x}}{\int_{\Omega_{2}} 1 \mathrm{dx}}
$$



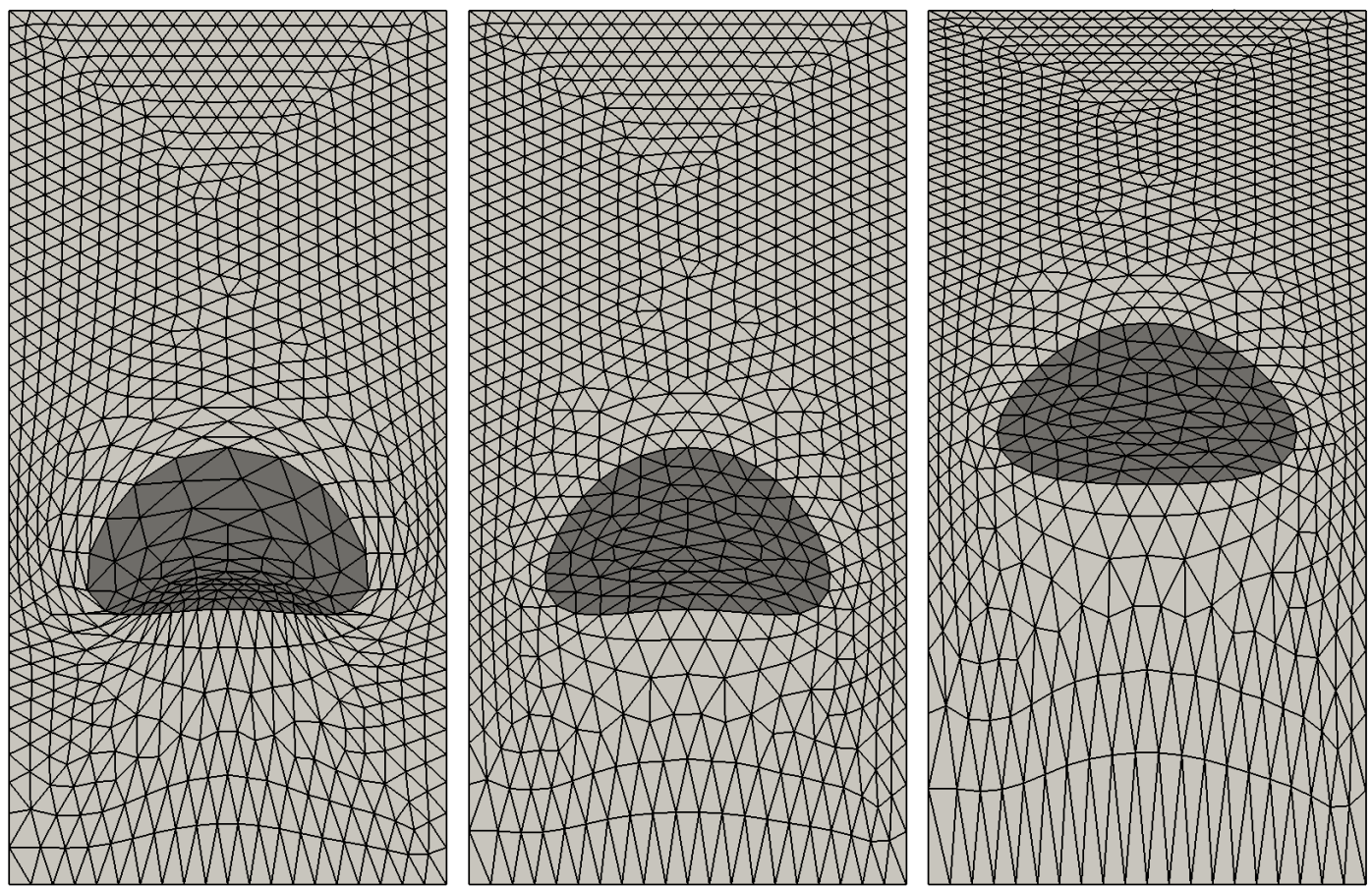

Figure 7. Mesh deformation on mesh M1 for Test Case 1 with different interface velocity update approaches. Left: approach (15) at $t=1.5$. Middle: approach (16) at $t=1.5$. Right: approach $(16)$ at $t=3$.
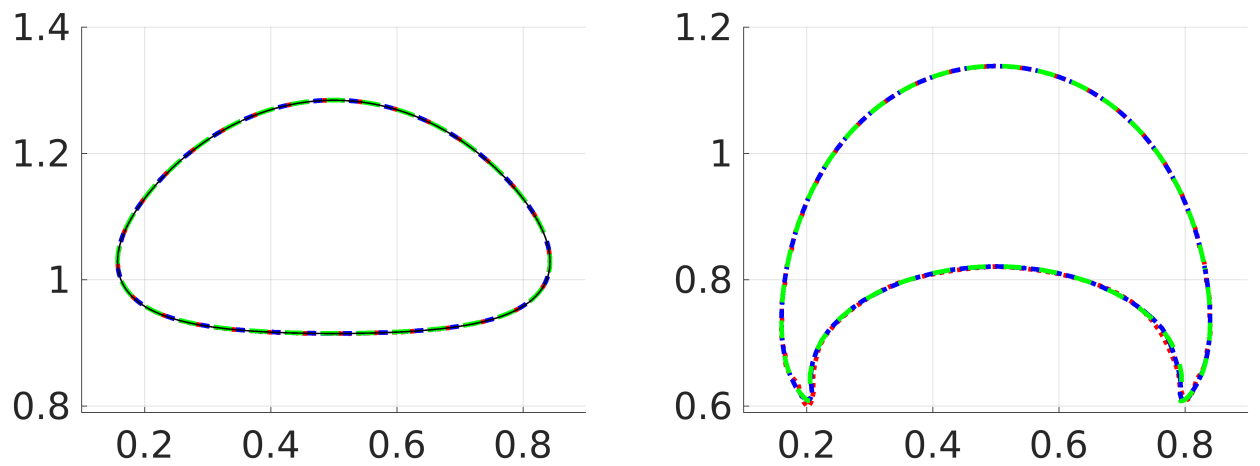

Figure 8. Bubble shapes at the final time. Left: Test Case 1 at time $t=3$. Right: Test Case 2 at time $t=2$. (M1: dotted red, M2: dashdotted blue, M3: dashed green. MooNMD reference data [29] (for Test Case 1): solid black.)

(ii) Rise velocity: the mean bubble velocity

$$
\mathbf{X}_{c}=\left(x_{c}, y_{c}\right)=\frac{\int_{\Omega_{2}} \boldsymbol{u} \mathrm{dx}}{\int_{\Omega_{2}} 1 \mathrm{dx}} .
$$

(iii) Circularity: the "degree of circularity":

$$
\not=\frac{P_{a}}{P_{b}}=\frac{\text { perimeter of area-equivalent circle }}{\text { perimeter of bubble }} .
$$

The time evolution of these benchmark quantities are shown in Fig. 10. Our results are in excellent agreement with the MooNMD reference data 29]. 

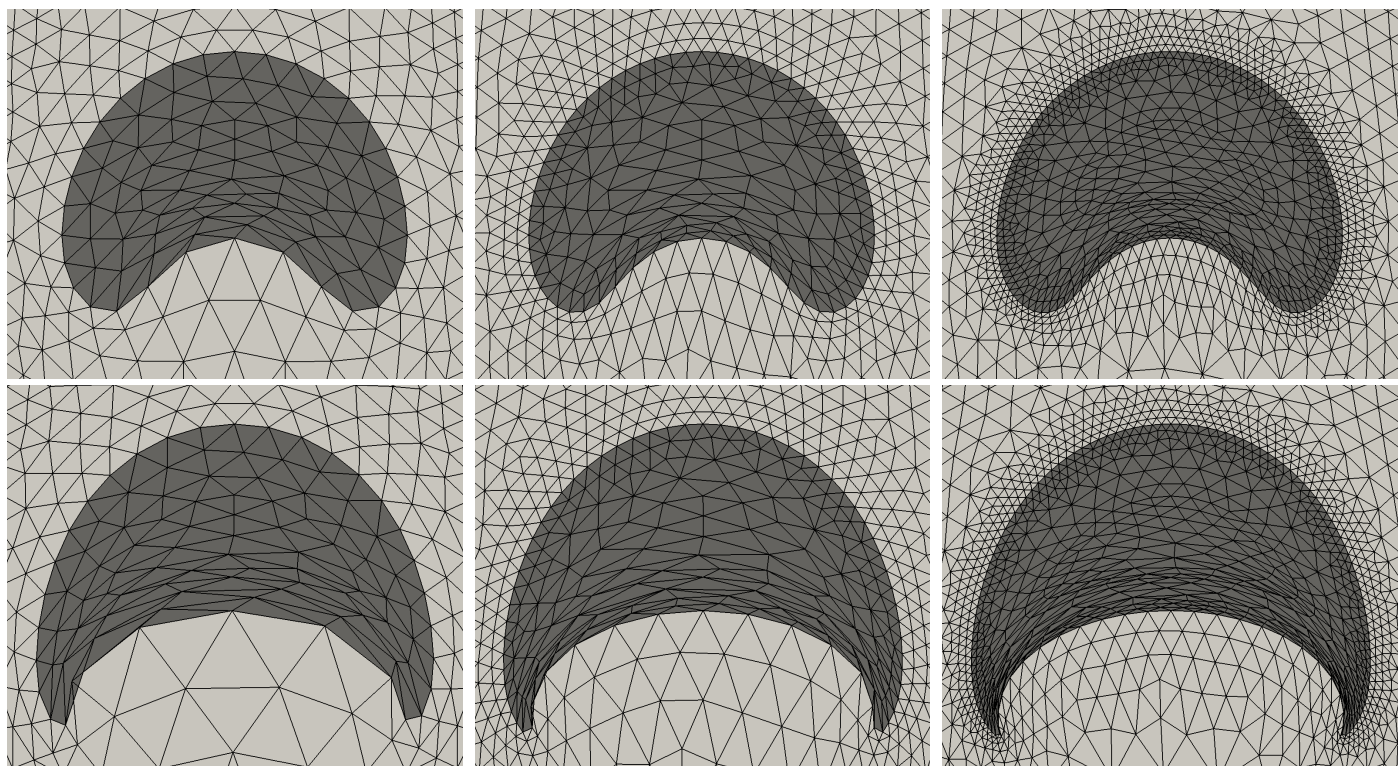

Figure 9. Zoom-in of the deformed mesh around $\Omega_{2}$. Left: M1. Middle: M2. Right: M3. Top: $t=1$. Bottom: $t=2$.

Finally, Table 4 and Table 5 show the time and values of the minimum circularity, maximum rise velocity, and maximum position of the center of mass achieved during the simulations for Test Case 1 and 2, respectively. Again, our results are in good agreement with the MooNMD reference data $[29$.

\begin{tabular}{lcccc}
\hline & M1 & M2 & M3 & MooNMD $[29]$ \\
\hline$\phi_{\min }$ & 0.90121 & 0.90123 & 0.90125 & 0.9013 \\
$\left.t\right|_{\phi=\phi_{\min }}$ & 1.9000 & 1.8969 & 1.8984 & 1.9000 \\
$V_{c, \max }$ & 0.24154 & 0.24161 & 0.24164 & 0.2417 \\
$\left.t\right|_{V_{c}=V_{c, \max }}$ & 0.91875 & 0.92188 & 0.92188 & 0.9239 \\
$y_{c}(t=3)$ & 1.0817 & 1.0817 & 1.0817 & 1.0817 \\
\hline
\end{tabular}

TABLE 4. Minimum circularity and maximum rise velocity, with corresponding incidence times, and the final position of the center of mass for Test Case 1.

\begin{tabular}{lcccc}
\hline & M1 & M2 & M3 & MooNMD $[29]$ \\
\hline$\phi_{\min }$ & 0.68784 & 0.69210 & 0.68922 & 0.6901 \\
$\left.t\right|_{\phi=\phi_{\min }}$ & 2.0000 & 2.0000 & 2.0000 & 2.0000 \\
$V_{c, \max }$ & 0.24978 & 0.25001 & 0.25016 & 0.2502 \\
$\left.t\right|_{V_{c}=V_{c, \max }}$ & 0.72500 & 0.73125 & 0.72969 & 0.7317 \\
$y_{c}(t=2)$ & 0.91473 & 0.91524 & 0.91541 & 0.9154 \\
\hline
\end{tabular}

TABLE 5. Minimum circularity and maximum rise velocity, with corresponding incidence times, and the final position of the center of mass for Test Case 2 (up to time $t=2)$. 

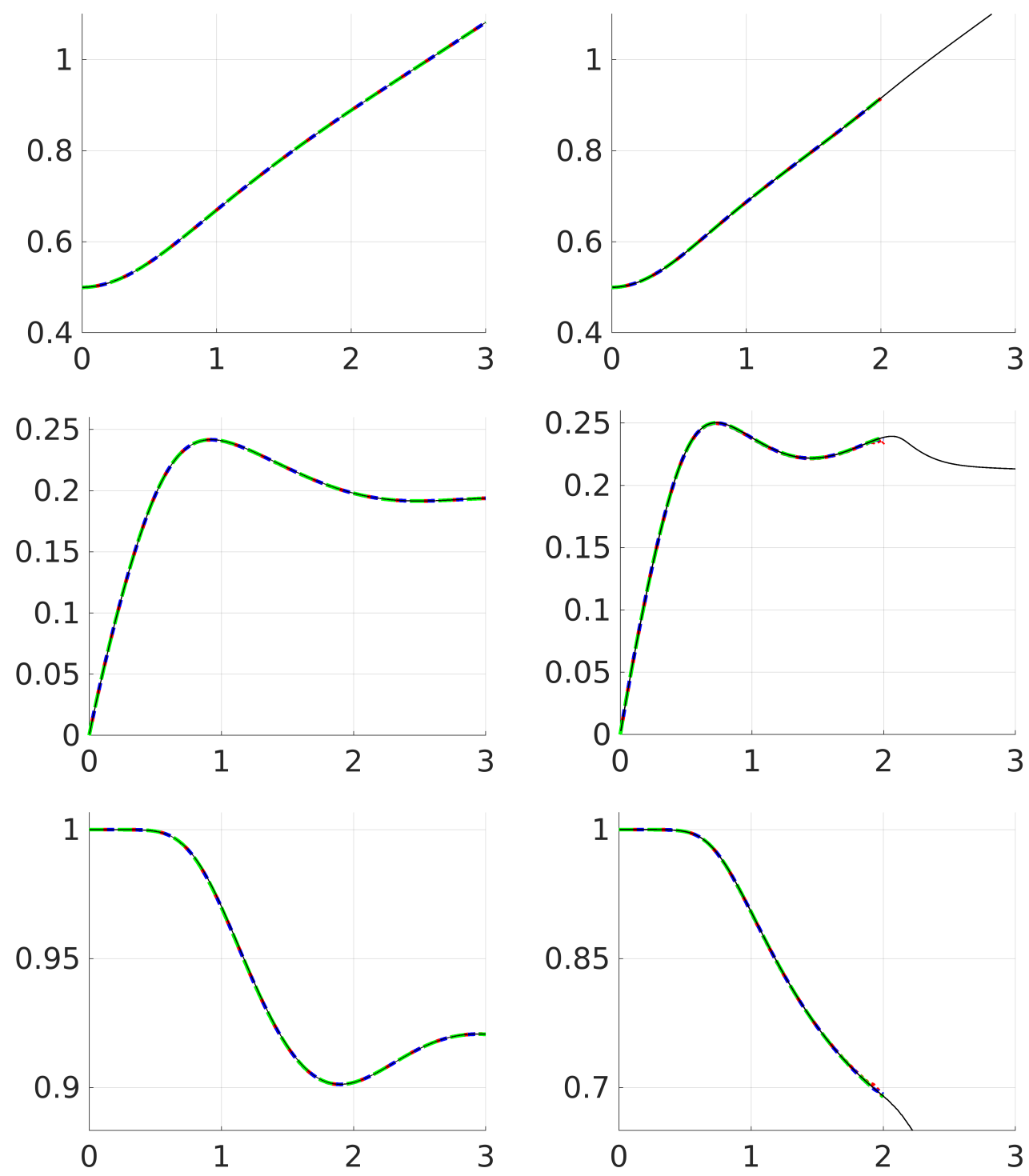

Figure 10. Time evolution of benchmark quantities. Left: Test Case 1. Right: Test Case 2. Top: center of mass. Middle: rise velocity. Bottom: circularity. (M1: dotted red, M2: dashdotted blue, M3: dashed green. MooNMD reference data 29]: solid black.)

\section{CONClusion And FUture WORK}

We have presented a novel ALE-TVNNS-HDG scheme for incompressible flow with moving boundaries and interfaces. Detailed theoretical analysis of the scheme consists of our ongoing work. We are also planning to investigate the extension of this scheme to fluid-structure interactions.

\section{REFERENCES}

[1] D. N. Arnold And F. Brezzi, Mixed and nonconforming finite element methods: implementation, postprocessing and error estimates, RAIRO Modél. Math. Anal. Numér., 19 (1985), pp. 7-32.

[2] U. M. Ascher, S. J. Ruuth, And B. T. R. Wetton, Implicit-explicit methods for time-dependent partial differential equations, SIAM J. Numer. Anal., 32 (1995), pp. 797-823. 
[3] J. Barrett, H. Garcke, And R. Nürnberg, A parametric finite element method for fourth order geometric evolution equations, J. Comput. Phys., 222 (2007), pp. 441-462.

[4] S. Basting, A. Quaini, S. ČAnić, And R. Glowinski, Extended ALE method for fluid-structure interaction problems with large structural displacements, J. Comput. Phys., 331 (2017), pp. 312-336.

[5] A. N. Brooks And T. J. R. Hughes, Streamline upwind/Petrov-Galerkin formulations for convection dominated flows with particular emphasis on the incompressible Navier-Stokes equations, Comput. Methods Appl. Mech. Engrg., 32 (1982), pp. 199-259. FENOMECH "81, Part I (Stuttgart, 1981).

[6] J. Chessa And T. Belytschko, An extended finite element method for two-phase fluids, Trans. ASME J. Appl. Mech., 70 (2003), pp. 10-17.

[7] B. CockBurn, Static condensation, hybridization, and the devising of the HDG methods, in Building bridges: connections and challenges in modern approaches to numerical partial differential equations, vol. 114 of Lect. Notes Comput. Sci. Eng., Springer, [Cham], 2016, pp. 129-177.

[8] B. Cockburn and J. Gopalakrishnan, A characterization of hybridized mixed methods for second order elliptic problems, SIAM J. Numer. Anal., 42 (2004), pp. 283-301.

[9] B. Cockburn, G. Kanschat, and D. Schötzau, A note on discontinuous Galerkin divergence-free solutions of the Navier-Stokes equations, J. Sci. Comput., 31 (2007), pp. 61-73.

[10] B. Cockburn And F.-J. Sayas, Divergence-conforming HDG methods for Stokes flows, Math. Comp., 83 (2014), pp. 1571-1598.

[11] J. Donea, S. Giuliani, And J. Halleux, An arbitrary lagrangian-eulerian finite element method for transient dynamic fluid-structure interactions, Comput. Methods Appl. Mech. Engrg., 33 (1982), pp. 689 - 723.

[12] J. Donea, A. Huerta, J.-P. Ponthot, And A. Rodriguez-Ferran, Arbitrary Lagrangian-Eulerian Methods, in Encyclopedia of Computational Mechanics, Volume 1: Fundamentals, chapter 14, E. Stein, R. De Borst, and T. J. R. Hughes, eds., John Wiley \& Sons, 2004.

[13] F. Duarte, R. Gormaz, And S. Natesan, Arbitrary Lagrangian-Eulerian method for Navier-Stokes equations with moving boundaries, Comput. Methods Appl. Mech. Engrg., 193 (2004), pp. 4819-4836.

[14] M. A. Fernández, J.-F. Gerbeau, and C. Grandmont, A projection semi-implicit scheme for the coupling of an elastic structure with an incompressible fluid, Internat. J. Numer. Methods Engrg., 69 (2007), pp. $794-821$.

[15] T.-P. Fries And T. Belytschko, The extended/generalized finite element method: an overview of the method and its applications, Internat. J. Numer. Methods Engrg., 84 (2010), pp. 253-304.

[16] B. Froehle And P.-O. Persson, Nonlinear Elasticity for Mesh Deformation with High-Order Discontinuous Galerkin Methods for the Navier-Stokes Equations on Deforming Domains, in Spectral and High Order Methods for Partial Differential Equations ICOSAHOM 2014, vol. 106, 2015, pp. 77-85.

[17] G. Fu, An explicit divergence-free DG method for incompressible flow, Comput. Methods Appl. Mech. Engrg., 345 (2019), pp. 502-517.

[18] — An explicit divergence-free DG method for incompressible magnetohydrodynamics, J. Sci. Comput., 79 (2019), pp. 1737-1752.

[19] G. FU, Y. JIN, AND W. QIU, Parameter-free superconvergent H(div)-conforming HDG methods for the Brinkman equations, IMA J. Numer. Anal., 39 (2019), pp. 957-982.

[20] S. Ganesan, A. Hahn, K. Simon, And L. Tobiska, ALE-FEM for two-phase and free surface flows with surfactants, in Transport processes at fluidic interfaces, Adv. Math. Fluid Mech., Birkhäuser/Springer, Cham, 2017, pp. 5-31.

[21] R. Glowinski, T. W. Pan, T. I. Hesla, D. D. Joseph, and J. PÉRiaux, A fictitious domain approach to the direct numerical simulation of incompressible viscous flow past moving rigid bodies: application to particulate flow, J. Comput. Phys., 169 (2001), pp. 363-426.

[22] S. Gross And A. Reusken, Numerical methods for two-phase incompressible flows, vol. 40 of Springer Series in Computational Mathematics, Springer-Verlag, Berlin, 2011.

[23] P. Hansbo, M. G. Larson, And S. Zahedi, Stabilized finite element approximation of the mean curvature vector on closed surfaces, SIAM J. Numer. Anal., 53 (2015), pp. 1806-1832.

[24] C.-J. HeINE, Isoparametric finite element approximation of curvature on hypersurfaces, Citeseer, (2004).

[25] T. J. Horvath And S. RheBergen, An exactly mass conserving space-time embedded-hybridized discontinuous Galerkin method for the Navier-Stokes equations on moving domains, arXiv:1910.08616 [math.NA].

[26] _ A locally conservative and energy-stable finite element method for the NavierStokes problem on timedependent domains, Int. J. Numer. Methods Fluids, (2019), pp. 519-532.

[27] T. J. R. Hughes, Multiscale phenomena: Green's functions, the Dirichlet-to-Neumann formulation, subgrid scale models, bubbles and the origins of stabilized methods, Comput. Methods Appl. Mech. Engrg., 127 (1995), pp. 387-401.

[28] T. J. R. Hughes, W. K. Liu, And T. K. Zimmermann, Lagrangian-Eulerian finite element formulation for incompressible viscous flows, Comput. Methods Appl. Mech. Engrg., 29 (1981), pp. 329-349. 
[29] S. Hysing, S. Turek, D. Kuzmin, N. Parolini, E. Burman, S. Ganesan, and L. Tobiska, Quantitative benchmark computations of two-dimensional bubble dynamics, Internat. J. Numer. Methods Fluids, 60 (2009), pp. $1259-1288$.

[30] D. Kamensky, M.-C. Hsu, Y. Yu, J. A. Evans, M. S. Sacks, and T. J. R. Hughes, Immersogeometric cardiovascular fluid-structure interaction analysis with divergence-conforming B-splines, Comput. Methods Appl. Mech. Engrg., 314 (2017), pp. 408-472.

[31] J. KönNö AND R. STEnBERG, Numerical computations with H(div)-finite elements for the Brinkman problem, Comput. Geosci., 16 (2012), pp. 139-158.

[32] E. V. Laitone, The second approximation to cnoidal and solitary waves, J. Fluid Mech., 9 (1960), pp. $430-444$.

[33] C. Lehrenfeld, Hybrid Discontinuous Galerkin methods for solving incompressible flow problems. Diploma Thesis, MathCCES/IGPM, RWTH Aachen, 2010.

[34] C. Lehrenfeld AND J. SchÖBERL, High order exactly divergence-free hybrid discontinuous Galerkin methods for unsteady incompressible flows, Comput. Methods Appl. Mech. Engrg., 307 (2016), pp. 339-361.

[35] Z. LI, The immersed interface method using a finite element formulation, Appl. Numer. Math., 27 (1998), pp. 253-267.

[36] A. Masud And T. J. R. Hughes, A space-time Galerkin/least-squares finite element formulation of the NavierStokes equations for moving domain problems, Comput. Methods Appl. Mech. Engrg., 146 (1997), pp. 91-126.

[37] R. Mittal and G. Iaccarino, Immersed boundary methods, Annu. Rev. Fluid Mech., 37 (2005), pp. $239-261$.

[38] M. Neunteufel, Advanced Numerical Methods for Fluid Structure Interaction. Diploma Thesis, TU Wien, 2017.

[39] N. C. Nguyen, J. Peraire, and B. Cockburn, A hybridizable discontinuous Galerkin method for Stokes flow, Comput. Methods Appl. Mech. Engrg., 199 (2010), pp. 582-597.

[40] F. NoBILE, Numerical approximation of fluid-structure interaction problems with application to haemodynamics, $\mathrm{PhD}$ thesis, École polytechnique fédérale de Lausanne, 2001.

[41] C. S. PESKIn, The immersed boundary method, Acta Numer., 11 (2002), pp. 479-517.

[42] A. QuAini, Algorithms for Fluid-Structure Interaction Problems Arising in Hemodynamics, PhD thesis, École polytechnique fédérale de Lausanne, 2009.

[43] B. Ramaswamy and M. Kawahara, Arbitrary Lagrangian-Eulerian finite element method for unsteady, convective, incompressible viscous free surface fluid flow, Int. J. Numer. Methods Fluids, 7 (1987), pp. 1053-1075.

[44] S. Rhebergen And G. N. Wells, A hybridizable discontinuous Galerkin method for the Navier-Stokes equations with pointwise divergence-free velocity field, J. Sci. Comput., 76 (2018), pp. 1484-1501.

[45] T. Richter, Fluid-structure interactions, vol. 118 of Lecture Notes in Computational Science and Engineering, Springer, Cham, 2017. Models, analysis and finite elements.

[46] J. SchöBerL, C++11 Implementation of Finite Elements in NGSolve, 2014. ASC Report 30/2014, Institute for Analysis and Scientific Computing, Vienna University of Technology.

[47] P. W. Schroeder, V. John, P. L. Lederer, C. Lehrenfeld, G. Lube, And J. SchöBerl, On reference solutions and the sensitivity of the 2D Kelvin-Helmholtz instability problem, Comput. Math. Appl., 77 (2019), pp. 1010-1028.

[48] P. W. Schroeder, C. Lehrenfeld, A. Linke, And G. Lube, Towards computable flows and robust estimates for inf-sup stable FEM applied to the time-dependent incompressible Navier-Stokes equations, SeMA J., 75 (2018), pp. 629-653.

[49] J. P. Sheldon, S. T. Miller, And J. S. PitT, A hybridizable discontinuous Galerkin method for modeling fluid-structure interaction, J. Comput. Phys., 326 (2016), pp. 91-114.

[50] T. E. Tezduyar, S. Aliabadi, And M. Behr, Enhanced-Discretization Interface-Capturing Technique (EDICT) for computation of unsteady flows with interfaces, Comput. Methods Appl. Mech. Engrg., 155 (1998), pp. $235-248$.

[51] T. E. Tezduyar, M. Behr, And J. Liou, A new strategy for finite element computations involving moving boundaries and interfaces-the deforming-spatial-domain/space-time procedure. I. The concept and the preliminary numerical tests, Comput. Methods Appl. Mech. Engrg., 94 (1992), pp. 339-351.

[52] T. E. Tezduyar, M. Behr, S. Mittal, And J. Liou, A new strategy for finite element computations involving moving boundaries and interfaces - the deforming-spatial-domain/space-time procedure. II. Computation of freesurface flows, two-liquid flows, and flows with drifting cylinders, Comput. Methods Appl. Mech. Engrg., 94 (1992), pp. 353-371.

[53] T. E. Tezduyar, K. Takizawa, And Y. Bazilevs, FluidStructure Interaction and Flows with Moving Boundaries and Interfaces, in Encyclopedia of Computational Mechanics Second Edition, Part 2 Fluids, E. Stein, R. De Borst, and T. J. R. Hughes, eds., John Wiley \& Sons, 2018.

[54] J. J. W. VAn Der Vegt And J. J. Sudirham, A space-time discontinuous Galerkin method for the timedependent Oseen equations, Appl. Numer. Math., 58 (2008), pp. 1892-1917. 
[55] T. M. van Opstal, J. Yan, C. Coley, J. A. Evans, T. Kvamsdal, and Y. Bazilevs, Isogeometric divergenceconforming variational multiscale formulation of incompressible turbulent flows, Comput. Methods Appl. Mech. Engrg., 316 (2017), pp. 859-879.

[56] T. WICK, Fluid-structure interactions using different mesh motion techniques, Comput. Struct., 89 (2011), pp. 1456-1467.

[57] S. Yirgit, M. SchäFer, AND M. Heck, Grid movement techniques and their influence on laminar fluid-structure interaction problems, J. Fluids Struct., 24 (2008), pp. 819-832.

[58] L. Zhang, A. Gerstenberger, X. Wang, and W. K. Liu, Immersed finite element method, Comput. Methods Appl. Mech. Engrg., 193 (2004), pp. 2051-2067.

Department of Applied and Computational Mathematics and Statistics, University of Notre Dame, USA.

E-mail address: gfu@nd.edu 